Pacific Journal of Mathematics

EXISTENCE OF STRONG SOLUTIONS FOR STOCHASTIC 


\section{EXISTENCE OF STRONG SOLUTIONS FOR STOCHASTIC DIFFERENTIAL EQUATIONS IN THE PLANE}

\section{J. YEH}

Let $B$ be the 2-parameter Brownian motion on $D=$ $[0, \infty] \times[0, \infty)$ and $Z$ be a 2-parameter stochastic process defined on the boundary $\partial D$ of $D$. Consider the non-Markovian stochastic differential system in 2-parameter

$$
\left\{\begin{array}{lll}
d X(s, t)=\alpha(s, t, X) d B(s, t)+\beta(s, t, X) d s d t & \text { for } & (s, t) \in D, \\
X(s, t)=Z(s, t) & \text { for } & (s, t) \in \partial D .
\end{array}\right.
$$

Under the assumption that the coefficients $\alpha$ and $\beta$ satisfy a Lipschitz condition and a growth condition and the assumption that $Z$ has continuous sample functions and locally bounded second moment on $\partial D$, it is shown in this paper that the differential system has a strong solution. Pathwise uniqueness of solution is established under the assumption of the Lipschitz condition.

o. Introduction. Recently several papers on stochastic integrals in the plane have appeared (see [2], [3], [9], [11] and [13]). In the present paper we treat stochastic differential equations in the plane. The domain of definition of the stochastic integrals and stochastic differential equations we consider is the positive quadrant $D=$ $[0, \infty) \times[0, \infty)$ in which a partial order $(s, t)<(u, v)$ for $s \leqq u$ and $t \leqq v$ is introduced. The object of our study is a stochastic differential equation of the type

$$
d X(s, t)=\alpha(s, t, X) d B(s, t)+\beta(s, t, X) d s d t
$$

or, to be precise,

$$
\begin{aligned}
X(s, t) & -X(s, 0)-X(0, t)+X(0,0) \\
& =\int_{[0, s] \times[0, t]} \alpha(u, v, X) d B(u, v) \int_{[0, s] \times[0, t]} \beta(u, v, X) d(u, v),
\end{aligned}
$$

where $B$ is a 2-parameter Brownian motion on a probability space $(\Omega, \mathfrak{F}, P)$ and the domain $D$. The precise defininions of $B$ and the two stochastic integrals appearing on the right side of (0.1) are given in $\S 2$. The case in which the coefficients of the stochastic differential equation depend on $X$ only to the extent that they depend on $X(s, t)$, i.e., the stochastic differential equation

$$
d X(s, t)=a(X(s, t)) d B(s, t)+b(X(s, t)) d s d t
$$

was treated by Cairoli [2]. The coefficients $\alpha$ and $\beta$ in our equation 
$(0,1)$ are real valued functions on $D \times W$ where $W$ is the space of all real valued continuous functions on $D$. We impose certain measurability conditions on $\alpha$ and $\beta$ so that $\alpha(s, t, X)$ and $\beta(s, t, X)$ depend only on that part of the sample function of $X$ which preceeds $(s, t)$ in the sense of the partial order $\prec$.

Let $\left(\Omega, \widetilde{\Im}, P ; \widetilde{\mho}_{s, t}\right)$ be a probability space with a family of sub- $\sigma$ algebras $\widetilde{\mho}_{s, t},(s, t) \in D$, satisfying the usual conditions (see Definition 1.1 in $\S 1)$. For brevity we call $\left(\Omega, \widetilde{\mho}, P ; \widetilde{\mho}_{s, t}\right)$ an equipped probability space. By an $\widetilde{\mho}_{s, t}$ Brownian motion we mean a 2-parameter Brownian motion on $\left(\Omega, \widetilde{\mho}, P\right.$; $\left.\widetilde{\mho}_{s, t}\right)$ and $D$ which is $\widetilde{\mho}_{s, t}$ adapted. In $\S 2$ we define stochastic integrals of square integrable stochastic processes with respect to an $\widetilde{\mho}_{s, t}$ Brownian motion and state some well-known facts about such stochastic integrals in a way suitable for our use in treating the stochastic differntial equation (0.1).

By a boundary stochastic process we mean a 2-parameter stochastic process whose domain of definition is the boundary $\partial D$ of $D$.

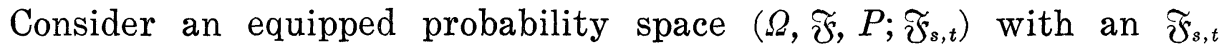
Brownian motion $B$ with $\partial B=0$ on it. In $\S 3$ we show that if the coefficients $\alpha$ and $\beta$ in (0.1) satisfy the Lipschitz condition (3.3) and the growth condition (3.4) in $\S 3$, then for every boundary stochastic process $Z$ which is $\widetilde{\mho}_{s, t}$ adapted and has continuous sample functions on $\partial D$ and bounded second moments on every bounded subset of $\partial D$ there exists a 2-parameter stcochastic process $X$ which is $\widetilde{\mho}_{s, t}$ adapted, has continuous sample functions on $D$ and bounded second moments on every bounded subset of $D$ and satisfies (0.1) and the boundary condition $\partial X=Z$. Strong solutions of stochastic differential equations were discussed in Liptzer and Shiryaev [7] and Watanabe [10]. In $\S 3$ we define strong solutions for stochastic differential equations in the plane. In Theorem 3.12 we show that under the assumption of the conditions (3.3) and (3.4) on the coefficients $\alpha$ and $\beta$ in (0.1) a strong solution exists. Regarding the uniqueness of the solution of (0.1), Theorem 3.8 shows that under the assumption of (3.3) on $\alpha$ and $\beta$, the boundary condition $\partial X=Z$ determines the solution $X$ of (0.1) almost surely.

1. Stochastic processes and Martingales in the plane. Throughout this paper the domain of definition of a stochastic process in the plane is the set $D=[0, \infty) \times[0, \infty)$. We write $\partial D$ for the boundary of $D$ as a subset of $(-\infty, \infty) \times(-\infty, \infty)$ relative to the Euclidean topology. In $D$ we introduce a partial order $\prec$ by writing

$$
(s, t) \prec\left(s^{\prime}, t^{\prime}\right) \text { when } s \leqq s^{\prime} \text { and } t \leqq t^{\prime} \text {. }
$$

We write

$$
(s, t) \prec \prec\left(s^{\prime}, t^{\prime}\right) \quad \text { when } \quad s<s^{\prime} \text { and } t<t^{\prime} \text {. }
$$


A transformation $\varphi$ of $D$ into a set with a partial order $\prec$ is said to be increasing if

$$
(s, t) \prec\left(s^{\prime}, t^{\prime}\right) \Longrightarrow \varphi(s, t) \prec \varphi\left(s^{\prime}, t^{\prime}\right) .
$$

A measurable transformation of a measurable space $\left(S_{1}, \mathfrak{A}_{1}\right)$ into another $\left(S_{2}, \mathfrak{A}_{2}\right)$ will be called an $\mathfrak{U}_{1} / \mathfrak{U}_{2}$ measurable transformation. We write $\mathfrak{B}(S)$ for the $\sigma$-algebra of Borel sets in a topological space $S$. For the Lebesque measures on $\left(\boldsymbol{R}^{k}, \mathfrak{B}\left(\boldsymbol{R}^{k}\right)\right)$ for $k=1,2, \cdots$ we use the generic notation $m_{L}$.

By a 2-parameter stochastic process on a probability space $(\Omega, \widetilde{F}, P)$ we mean a transformation $X$ of $D \times \Omega$ into $\boldsymbol{R}$ in which $(s, t, \cdot)$ is $\mathfrak{F} / \mathfrak{B}(\boldsymbol{R})$ measurable for every $(s, t) \in D$. At times we write $X_{s, t}$ for $X(s, t, \cdot)$. By a boundary stochastic process on $(\Omega, \widetilde{F}, P)$ we mean a transformation $Z$ of $\partial D \times \Omega$ into $\boldsymbol{R}$ in which $Z(s, t, \cdot)$ is $\mathfrak{F} / \mathfrak{B}(\boldsymbol{R})$ measurable for evey $(s, t) \in \partial D$. When $X$ is a 2-parameter stochastic process its restriction to $\partial D \times \Omega$ is a boundary stochastic process and we write $\partial X$ for it. For two random variables $\xi$ and $\eta$ on $(\Omega, \widetilde{F}, P)$ we write $\xi=\eta$ when $\xi(\omega)=\eta(\omega)$ for a.e., $\omega \in \Omega$. We say that two 2-parameter stochastic processes $X$ and $Y$ are equivalent and write $X=Y$ when $X(\cdot, \cdot, \omega)=Y(\cdot, \cdot, \omega)$ for a.e. $\omega \in \Omega$. The equality of two boundary stochastic processes is defined in the same way.

Definition 1.1. Let $\left\{\widetilde{\mho}_{s, t},(s, t) \in D\right\}$ be a system of sub- $\sigma$-algebras of $\mathfrak{F}$ in a probability space $(\Omega, \mathfrak{F}, P)$. We call

$$
\left(\Omega, \mathfrak{F}, P ; \mathfrak{F}_{s, t}\right) \equiv\left(\Omega, \widetilde{F}, P:\left\{\mathfrak{F}_{s, t},(s, t) \in D\right\}\right)
$$

an equipped probability space if

$1^{\circ}(\Omega, \widetilde{F}, P)$ is a complete probability measure space,

$2^{\circ}\left\{\widetilde{F}_{s, t},(s, t) \in D\right\}$ is an increasing system in the sense that $(s, t) \prec\left(s^{\prime}, t^{\prime}\right)$ implies $\widetilde{\mho}_{s, t} \subset \widetilde{\mho}_{s^{\prime}, t^{\prime}}$,

$3^{\circ} \quad \widetilde{F}_{0,0}$ contatins all the null sets in $(\Omega, \mathfrak{F}, P)$,

$4^{\circ}\left\{\widetilde{\mho}_{s, t},(s, t) \in D\right\}$ is a right continuous system in the sense that for every $(s, t) \in D$

$$
\widetilde{\mho}_{s, t}=\bigcap_{(s, t)<<\left(s^{\prime}, t^{\prime}\right)} \widetilde{\mho}_{s^{\prime}, t^{\prime}},
$$

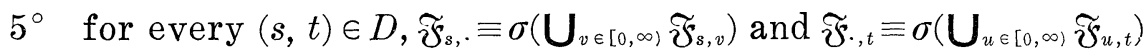
are conditionally independent relative to $\widetilde{F}_{s, t}$.

A 2-parameter stochastic process $X$ on an equipped probability space $\left(\Omega, \widetilde{\mho}, P ; \widetilde{\mho}_{s, t}\right)$ is said to be $\widetilde{\mho}_{s, t}$ adapted if for every $(s, t) \in D$, $X(s, t, \cdot)$ is $\widetilde{\mho}_{s, t} / \mathfrak{B}(\boldsymbol{R})$ measurable. Similarly a boundary stochastic process $Z$ is said to be $\widetilde{\mho}_{s, t}$ adapted if for every $(s, t) \in \partial D, Z(s, t, \cdot)$ is $\widetilde{\mho}_{s, t} / \mathfrak{B}(\boldsymbol{R})$ measurable. 
DEFINITION 1.2. A 2-parameter stochastic process $X$ on an equipped probability space $\left(\Omega, \widetilde{\mho}, P\right.$; $\left.\Im_{s, t}\right)$ is called a 2-parameter martingale relative to $\left\{\widetilde{F}_{s, t},(s, t) \in D\right\}$ if

$1^{\circ} X$ is $\widetilde{\mho}_{s, t}$ adapted,

$2^{\circ} \quad E\left[\left|X_{s, t}\right|\right]<\infty$ for every $(s, t) \in D$,

$3^{\circ} \quad \boldsymbol{E}\left[X_{s^{\prime}, t^{\prime}} \mid \mathfrak{\mho}_{s, t}\right]=X_{s, t}$ whenever $(s, t) \prec\left(s^{\prime}, t^{\prime}\right)$.

A 2-parameter martingale $X$ is said to be square integrable if $4^{\circ} \quad \boldsymbol{E}\left[X_{s, t}^{2}\right] \prec \infty$ for every $(s, t) \in D$.

It is said to be right continuous if

$5^{\circ} \lim _{\substack{\left(s^{\prime}, t^{\prime}\right)(s, t) \\(s, t)<\left(s^{\prime}, t^{\prime}\right)}} X_{s^{\prime}, t^{\prime}}(\omega)=X_{s, t}(\omega)$ for all $(s, t) \in D$ for $(s, t) \prec\left(s^{\prime}, t^{\prime}\right)$ a.e. $\omega \in \Omega$.

DEFINITION 1.3. Given an equipped probability space $\left(\Omega, \mathfrak{F}, P ; \mathfrak{F}_{s, t}\right)$, we write $\mathfrak{M}_{2}\left(\widetilde{\mho}_{s, t}\right)$ for the linear space of equivalence classes of right continuous square integrable martingales $X$ on $\left(\Omega, \widetilde{F}, P ; \mathfrak{F}_{s, t}\right)$ with $\partial X=0$. We write $\mathfrak{M}_{2}^{c}\left(\Im_{s, t}\right)$ for the collection of $X \in \mathfrak{M}_{2}\left(\mathfrak{\mho}_{s, t}\right)$ such that $(\cdot, \cdot, \omega)$ is continuous on $D$ for a.e. $\omega \in \Omega$.

Definition 1.4. For $X \in \mathfrak{M}_{2}\left(\widetilde{\mho}_{s, t}\right)$, we write

$$
\begin{gathered}
|X|_{(s, t)}=\left\{E\left[X_{s, t}^{2}\right]\right]^{1 / 2} \text { for }(s, t) \in D, \\
|X|_{T}=|X|_{(T, T)} \text { for } T \in[0, \infty), \\
|X|=\sum_{k=1}^{\infty} 2^{-k}\left\{|X|_{k} \wedge 1\right\} .
\end{gathered}
$$

Proposition 1.5. $\mathfrak{M}_{2}\left(\mathfrak{F}_{s, t}\right)$ is a Banach space relative to the norm $|\cdot|$ and $\mathfrak{M}_{2}^{c}\left(\mathfrak{\mho}_{s, t}\right)$ is a closed linear subspace of $\mathfrak{M}_{2}\left(\widetilde{\mho}_{s, t}\right)$.

Proposition 1.6. If a sequence $\left\{X^{(n)}, n=1,2, \cdots\right\}$ in $\mathfrak{M}_{2}\left(\mathfrak{F}_{s, t}\right)$ converges to some $X$ in $\mathfrak{M}_{2}\left(\mathfrak{\mho}_{s, t}\right)$, then for every $T>0$

$$
P-\lim _{n \rightarrow \infty}\left\{\sup _{(s, t)<(T, T)}\left|X_{s t}^{(n)}-X_{s, t}\right|\right\}=0
$$

and there exist a subsequence $\{m\}$ of $\{n\}$ and a null set $N$ in $(\Omega, \Im, P)$ such that

$$
\lim _{m \rightarrow \infty} X^{(m)}(s, t, \omega)=X(s, t, \omega)
$$

uniformly on every bounded subset of $D$ when $\omega \in N^{c}$.

2. Stochastic integrals in the plane.

DeFinition 2.1. Given an equipped probability space $\left(\Omega, \mathfrak{F}, P ; \mathfrak{F}_{s, t}\right)$, by an $\mathfrak{\mho}_{s, t}$ Brownian motion on $\left(\Omega, \mathfrak{F}, P ; \mathfrak{\mho}_{s, t}\right)$ we mean a 2-parameter stochastic process $B$ on $(\Omega, \Im, P)$ and $D$ which satisfies the following 
conditions:

$1^{\circ}$ Every sample function of $B$ is continuous on $D$.

$2^{\circ} \quad$ For every finite rectangle of the type $\Delta=\left(s^{\prime}, s^{\prime \prime}\right] \times\left(t^{\prime}, t^{\prime \prime}\right] \subset D$ the random variable $B(\Delta)$ defined by

$$
B(\Delta)=B\left(s^{\prime \prime}, t^{\prime \prime}\right)-B\left(s^{\prime}, t^{\prime \prime}\right)-B\left(s^{\prime \prime}, t^{\prime}\right)+B\left(s^{\prime}, t^{\prime}\right)
$$

is destributed by $N\left(0,\left(s^{\prime \prime}-s^{\prime}\right)\left(t^{\prime \prime}-t^{\prime}\right)\right)$.

$3^{\circ}$ For every finite collection $\left\{\Delta_{1}, \cdots, \Delta_{n}\right\}$ of disjoint rectangles of the type above, the system of random variables $\left\{B\left(\Delta_{1}\right), \cdots, B\left(\Delta_{n}\right)\right\}$ in an independent system.

$4^{\circ} \quad B$ is an $\widetilde{\mho}_{s, t}$ adapted stochastic process.

We write $\partial B=0$ for a 2-parameter Brownian motion $B$ when every sample function of $B$ vaninshes on $\partial D$.

DEFINITION 2.2. A 2-parameter stochastic process $X$ on a probability space $(\Omega, \Im, P)$ and $D$ is called a measurable process if it is $\sigma(\mathfrak{B}(D) \times \mathfrak{F}) / \mathfrak{B}(\boldsymbol{R})$ measurable. A 2-parameter stochastic process $X$ on an equipped probability space $\left(\Omega, \mathfrak{F}, P ; \mathfrak{F}_{s, t}\right)$ and $D$ is said to be progressively measurable if for every $(s, t) \in D$ the restriction of $X$ to $[0, s] \times[0, t] \times \Omega$ is $\sigma\left(\mathfrak{B}([0, s] \times[0, t]) \times \widetilde{F}_{s, t}\right) / \mathfrak{B}(\boldsymbol{R})$ measurable.

Proposition 2.3. Let $X$ be an $\widetilde{\mho}_{s, t}$ adapted 2-parameter stochastic process on an equipped probability space $\left(\Omega, \widetilde{F}, P ; \mathfrak{F}_{s, t}\right)$. If every sample function of $X$ is right continuous i.e., for every $\omega \in \Omega$

$$
\lim _{\substack{(u) v)(s t) \\(s t)<(u v)}} X(u, v, \omega)=X(s, t, \omega) \quad \text { for } \quad(s, t) \in D,
$$

then $X$ is progressively measurable. The same holds when every sample function of $X$ is left continuous.

Definition 2.4. By $\mathfrak{R}_{p}\left(\mathfrak{F}_{s, t}\right), p \geqq 1$, we mean the linear space of equivalence classes of 2-parameter stochastic process $\Phi$ on an equipped probability space $\left(\Omega, \mathfrak{F}, P ; \mathfrak{F}_{s, t}\right)$ and $D$ which satisfy the following conditions:

$1^{\circ} \Phi$ is $\widetilde{\mho}_{s, t}$ adapted.

$2^{\circ} \Phi$ is a measurable process.

$3^{\circ}$ For every $T>0$

$$
\|\Phi\|_{p, T} \equiv \boldsymbol{E}\left[\left.\int_{[0, T] \times[0, T]}|\Phi(s, t, \cdot)|\right|^{p} m_{L}(d(s, t))\right]^{1 / p}<\infty .
$$

For $\Phi \in \mathfrak{R}_{p}\left(\widetilde{\mho}_{s, t}\right)$ we define

$$
\|\Phi\|_{p}=\sum_{k=1}^{\infty} 2^{-k}\left\{\|\Phi\|_{p, k} \wedge 1\right\},
$$


and for $(s, t) \in D$ we define

$$
\|\Phi\|_{p,(s, t)}=E\left[\int_{[0, s] \times[0, t]}|\Phi(u, v, \cdot)|{ }^{p} m_{L}(d(s, t))\right]^{1 / p} .
$$

It is easy to verify that $\|\cdot\|_{p}$ is a norm on $\mathfrak{Z}_{p}\left(\mathfrak{F}_{s, t}\right)$ and $\mathfrak{R}_{p}\left(\mathfrak{\mho}_{s, t}\right)$ is a Banach space relative to this norm.

Proposition 2.5. Consider the space $\mathfrak{R}_{p}\left(\mathfrak{F}_{s, t}\right), p \geqq 1$, on an equipped probability space $\left(\Omega, \widetilde{\mho}, P ; \mathfrak{\mho}_{s, t}\right)$. Every member of $\mathfrak{R}_{p}\left(\mathfrak{\mho}_{s, t}\right)$ has a progressively measurable version. In fact if $\Phi$ is a 2-parameter stochastic process on $\left(\Omega, \widetilde{\mho}, P ; \Im_{s, t}\right)$ which satisfies the conditions $1^{\circ}$, $2^{\circ}, 3^{\circ}$ of Definition 2.4 , then there exists a null set $N$ in $(\Omega, \widetilde{F}, P$ ) such that if we define

$$
\Psi(s, t, \omega)=\left\{\begin{aligned}
\lim \sup _{h \downarrow 0} h^{-2} \int_{[s-h, s] \times[t-h, t]} \Phi(u, v, \omega) m_{L}(d(u, v)) \\
\text { for } \quad(s, t) \in D \text { and } \omega \in N^{c}, \\
0 \quad \text { for } \quad(s, t) \in D \quad \text { and } \quad \omega \in N,
\end{aligned}\right.
$$

then $\Psi$ satisfies $1^{\circ}, 2^{\circ}, 3^{\circ}$ of Definition 2.4, and is progressively measurable and $\Psi=\Phi$ i.e., $\Psi(\cdot, \cdot, \omega)=\Phi(\cdot, \cdot, \omega)$ for a.e. $\omega \in \Omega$.

DEFINITION 2.6. By $\mathfrak{R}_{0}\left(\mathfrak{\mho}_{s, t}\right)$ we mean the linear space of equivalence classes of 2-parameter stochastic processes $\Phi$ on an equipped probability space $\left(\Omega, \mathfrak{F}, P ; \mathfrak{F}_{s, t}\right)$ and $D$ which satisfies the following conditions:

$1^{\circ} \Phi$ is $\widetilde{\mho}_{s, t}$ adapted.

$2^{\circ} \Phi$ is a measurable process.

$3^{\circ} \Phi$ is bounded in the sense that there exists $M>0$ such that

$$
|\Phi(s, t, \omega)| \leqq M \text { for }(s, t) \in D \text { and a.e. } \omega \in \Omega .
$$

$4^{\circ}$ There exist $0=s_{0}^{*} \prec s_{1}^{*} \prec \cdots$ with $\lim _{i \rightarrow \infty} s_{i}^{*}=\infty$ and $0=$ $t_{0}^{*} \prec t_{1}^{*} \prec \cdots$ with $\lim _{j \rightarrow \infty} t_{j}^{*}=\infty$ such that

$$
\begin{aligned}
\Phi(s, t, \omega)=\Phi\left(s_{2}^{*}, t_{j}^{*}, \omega\right) \text { for } \quad(s, t) \in\left[s_{i}^{*}, s_{i+1}^{*}\right) \times\left[t_{j}^{*}, t_{j+1}^{*}\right), \\
\\
i, j=0,1,2, \cdots \text { for a.e. } \omega \in \Omega .
\end{aligned}
$$

Clearly $\mathbb{S}_{0}\left(\mathfrak{F}_{s, t}\right) \subset \mathfrak{R}_{p}\left(\mathfrak{\digamma}_{s, t}\right)$ for $p \geqq 1$.

Proposition 2.7. For every $p \geqq 1, \mathbb{\Omega}_{0}\left(\mathfrak{F}_{s, t}\right)$ is dense in $\mathbb{R}_{p}\left(\mathfrak{F}_{s, t}\right)$ relative to the metric associated with $\|\cdot\|_{p}$, i.e., for every $\Phi \in \mathbb{R}_{p}\left(\mathfrak{F}_{s, t}\right)$ there exists a sequence $\left\{\Phi_{n}, n=1,2, \cdots\right\}$ in $\mathfrak{L}_{0}\left(\mathfrak{F}_{s, t}\right)$ such that $\lim _{n \rightarrow \infty}\left\|\Phi_{n}-\Phi\right\|_{p}=0$.

DEFINITION 2.8. Let $B$ be an $\widetilde{\mho}_{s, t}$ Brownian motion on an equipped 
probability space $\left(\Omega, \widetilde{\mho}, P\right.$; $\left.\widetilde{\mho}_{s, t}\right)$ and let $\Phi$ be a 2 -parameter stochastic process of the class $\mathfrak{R}_{0}\left(\mathfrak{\mho}_{s, t}\right)$ represented as

$$
\begin{gathered}
\Phi(s, t, \omega)=\Phi\left(s_{i}^{*}, t_{j}^{*}, \omega\right) \text { for } \quad(s, t) \in\left[s_{i}^{*}, s_{i+1}^{*}\right) \times\left[t_{i}^{*}, t_{j+1}^{*}\right), \\
i, j=0,1,2, \cdots \text { and } \omega \in N^{c},
\end{gathered}
$$

where $N$ is a null set in $(\Omega, \Im, P)$. The stochastic integral $I(\Phi)$ of $\Phi$ with respect to $B$ is a 2-parameter stochastic process on $\left(\Omega, \widetilde{\mho}, P ; \mathfrak{\mho}_{s, t}\right)$ and $D$ defined as follows:

For $(s, t) \in D$, say, $(s, t) \in\left[s_{m-1}^{*}, s_{m}^{*}\right) \times\left[t_{n-1}^{*}, t_{n}^{*}\right)$ let

$$
\left\{\begin{array}{l}
s_{i}=s_{i}^{*} \text { for } i=0,1, \cdots, m-1 \text { and } s_{m}=s \\
t_{j}=t_{j}^{*} \text { for } j=0,1, \cdots, n-1 \text { and } t_{n}=t
\end{array}\right.
$$

and

$$
\left\{\begin{aligned}
& \varphi_{i, j}(\omega)=\Phi\left(s_{i}, t_{j}, \omega\right) \text { for } i=0,1, \cdots, m ; \\
& j=1,2, \cdots, n \text { and } \omega \in N^{c}, \\
& \beta_{i, j}(\omega)=B\left(\left(s_{\imath-1}, s_{i}\right] \times\left(t_{j-1}, t_{j}\right]\right)(\omega) \\
& \text { for } i=1,2, \cdots, m ; \\
& j=1,2, \cdots, n \text { and } \omega \in \Omega .
\end{aligned}\right.
$$

We define

$$
I(\Phi)(s, t, \omega)= \begin{cases}\sum_{i=1}^{m} \sum_{j=1}^{n} \varphi_{i-1, j-1}(\omega) \beta_{i, j}(\omega) & \text { for } \omega \in N^{c} \\ 0 & \text { for } \omega \in N\end{cases}
$$

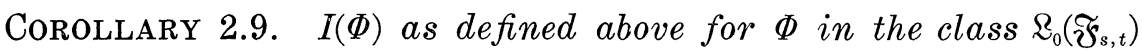
has the following properties:

(1) $I(\Phi)$ does not depend on the representation (1) of $\Phi$ in Definition 2.8. Also, if $\Phi$ and $\Psi$ are equivalent so are $I(\Phi)$ and $I(\Psi)$.

(2) $I(\Phi)$ is an $\widetilde{\mho}_{s, t}$ adapted process.

(3) Every sample function of $I(\Phi)$ is continuous on $D$.

(4) $\boldsymbol{E}\left[|(\Phi)(s, t, \cdot)|^{2}\right]<\infty$ from every $(s, t) \in D$ (from the boundedness of $\Phi)$.

(5) $\partial[I(\Phi)]=0$.

Proposition 2.10. $I(\Phi)$ as defined in Definition 2.8 for $\Phi$ in the class $\mathfrak{R}_{0}\left(\mathfrak{\mho}_{s, t}\right)$ is a 2-parameter martingale of the class $\mathfrak{M}_{2}^{c}\left(\mathfrak{\mho}_{s, t}\right)$.

Proposition 2.11. Let $I(\Phi)$ be as in Definition 2.8 for $\Phi$ in the class $\mathfrak{\Omega}_{0}\left(\mathfrak{\mho}_{s, t}\right)$. Then

$$
|I(\Phi)|_{(s, t)}=\|\Phi\|_{2,(s, t)} \text { for every } \quad(s, t) \in D
$$

so that in particular 


$$
\mid I(\Phi)]_{i}^{l_{T}}=\|\Phi\|_{2, T} \quad \text { for every } \quad T>0
$$

and

$$
|I(\Phi)|=\|\Phi\|_{2} .
$$

Let $\Phi$ be a 2-parameter stochastic process of the class $\Omega_{2}\left(\widetilde{\mho}_{s, t}\right)$ and let $B$ be an $\widetilde{F}_{s, t}$ Brownian motion on an equipped probability space $\left(\Omega, \widetilde{\mho}, P ; \mathfrak{\mho}_{s, t}\right)$. According to Proposition 2.7 there exists a sequence $\left\{\Phi_{n}, n=1,2, \cdots\right\}$ of 2-parameter stochastic processes of the class $\mathfrak{R}_{0}\left(\widetilde{\mho}_{s, t}\right)$ satisfying the condition $\lim _{n \rightarrow \infty}\left\|\Phi_{n}-\Phi\right\|_{2}=0$. According to Proposition 2.10 the stochastic integral $I\left(\Phi_{n}\right)$ of $\Phi_{n}$ with respect to $B$ is a 2-parameter martingale of the class $\mathfrak{M}_{2}\left(\widetilde{\mho}_{s, t}\right)$ and according to Proposition $2.11\left|I\left(\Phi_{m}\right)-I\left(\Phi_{n}\right)\right|=\left\|\Phi_{m}-\Phi_{n}\right\|_{2}$ so that $\left\{I\left(\Phi_{n}\right), \quad n=\right.$ $1,2, \cdots\}$ is a Cauchy sequence in $\mathfrak{M}_{2}\left(\widetilde{\mho}_{s, t}\right)$. Since $\mathfrak{M}_{2}^{c}\left(\widetilde{\mho}_{s, t}\right.$ is a complete metric space by Proposition 1.5, there exists a 2-parameter

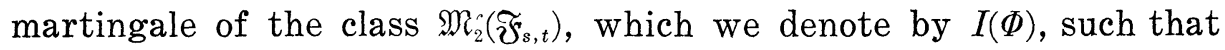

$$
\lim _{n \rightarrow \infty}\left|I\left(\Phi_{n}\right)-I(\Phi)\right|=0 .
$$

Observe that if $\left\{\Phi_{n}^{\prime}, n=1,2, \cdots\right\}$ is another sequence of 2-parameter stochastic processes of the class $\mathfrak{Z}_{0}\left(\widetilde{\mho}_{s, t}\right)$ such that $\lim _{n \rightarrow \infty}\left\|\Phi_{n}^{\prime}-\Phi\right\|_{2}=0$ and if $I\left(\Phi^{\prime}\right)$ is a 2-parameter martingale of the class $\mathfrak{M}_{2}^{r}\left(\Im_{s, t}\right)$ such that $\lim _{n \rightarrow \infty}\left|I\left(\Phi_{n}^{\prime}\right)-I\left(\Phi^{\prime}\right)\right|=0$ then by considering the sequence $\left\{\Phi_{1}, \Phi_{1}^{\prime}, \Phi_{2}, \Phi_{2}^{\prime}, \cdots\right\}$ we conclude that $I(\Phi)=I\left(\Phi^{\prime}\right)$ in $\mathfrak{M}_{2}^{\prime}\left(\mathfrak{\mho}_{s, t}\right)$ i.e., the two 2-parameter stochastic processes $I(\Phi)$ and $I\left(\Phi^{\prime}\right)$ are equivalent.

According to Definition 2.8 every sample function of $I\left(\Phi_{n}\right)$ is continuous on $D$. By Proposition 1.6 there exists a null set $N$ in

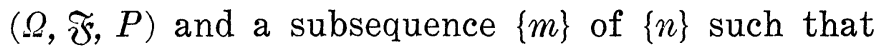

$$
\lim _{m \rightarrow \infty} I\left(\Phi_{m}\right)(s, t, \omega)=I(\Phi)(s, t, \omega)
$$

uniformly on every bounded subset of $D$ when $\omega \in N^{c}$ so that $I(\Phi)(\cdot, \cdot, \omega)$ is continuous on $D$ for $\omega \in N^{c}$. Let us define

$$
I(\Phi)(s, t, \omega)=0 \text { for } \quad(s, t) \in D \quad \text { when } \omega \in N .
$$

Then every sample function of $I(\Phi)$ is continuous on $D$.

DEFinition 2.12. By the stochastic integral of a 2-parameter stochastic process $\Phi$ of the class $\Omega_{2}\left(\widetilde{F}_{s, t}\right)$ with respect to an $\mathfrak{F}_{s, t}$ Brownian motion $B$ on an equipped probability space $\left(\Omega, \Im_{,}, P ; \widetilde{F}_{s, t}\right)$ we mean the 2-parameter martingale $I(\Phi)$ of the class $\mathfrak{M}_{2}\left(\Im_{s, t}\right)$ defined by (2.1), (2.2) and (2.3). We also use the notation

$$
\int_{[0, s] \times[0, t]} \Phi(u, v) d B(u, v) \text { for } I(\Phi)(s, t, \cdot) \text { for }(s, t) \in D .
$$


Note that from $\lim _{n \rightarrow \infty}\left\|\Phi_{n}\right\|_{2}=\|\Phi\|_{2}, \lim _{n \rightarrow \infty}\left|I\left(\Phi_{n}\right)\right|=|I(\Phi)|$ and $\left\|\Phi_{n}\right\|_{2}=\left|I\left(\Phi_{n}\right)\right|$ for $n=1,2, \cdots$, we have

$$
|I(\Phi)|=\|\Phi\|_{2} .
$$

This implies

$$
|I(\Phi)|_{(s, t)}=\|\Phi\|_{2,(s, t)} \text { for every }(s, t) \in D .
$$

Given an equipped probability $\operatorname{space}\left(\Omega, \mathfrak{F}, P ; \mathfrak{\mho}_{s, t}\right)$, let $\Psi$ be a 2 parameter stochastic process of the class $\mathfrak{R}_{1}\left(\mathfrak{\mho}_{s, t}\right)$. By Proposition 2.5 we assume that $\Psi$ is a progressively measurable version. By $3^{\circ}$ of Definition 2.4 there exists a null set $N$ in $(\Omega, \mathfrak{F}, P)$ such that

$$
\int_{[0, T] \times[0, T]}|\Psi(s, t, \omega)|\left(m_{L}(d(s, t))\right)<\infty \text { for all } T>0 \text { when } \omega \in N^{c} \text {. }
$$

Let us define a real valued function $J(\Psi)$ on $D \times \Omega$ by

$$
J(\Psi)(s, t, \omega)=\left\{\begin{aligned}
\int_{[0, s] \times[0, t]} \Psi(u, v, \omega) m_{L}(d(u, v)) \\
\text { for }(s, t) \in D \text { when } \omega \in N^{c}, \\
0 \quad \text { for }(s, t) \in D \text { when } \omega \in N .
\end{aligned}\right.
$$

The measurability of $\Psi$ and the fact that $N \in \mathfrak{F}$ imply that $J(\Psi)$ is a

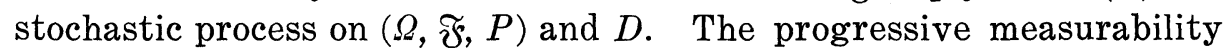
of $\Psi$ and the fact that $N \in \widetilde{\mho}_{s, t}$ for every $(s, t) \in D$ imply that $J(\Psi)$ is an $\mathfrak{\mho}_{s, t}$ adapted process on $\left(\Omega, \mathfrak{F}, P ; \mathfrak{\Im}_{s, t}\right)$. Also by $(2.7)$ every sample function of $J(\Psi)$ is continuous.

Definition 2.13. By the stochastic integral of a 2-parameter stochastic process $\Psi$ of the class $\mathfrak{R}_{1}\left(\widetilde{\mho}_{s, t}\right)$ with respect to the Lebesgue measure we mean the $\widetilde{\mho}_{s, t}$ adapted 2-parameter stochastic process $J(\Psi)$ on $\left(\Omega, \mathfrak{F}, P ; \mathfrak{F}_{s, t}\right)$ defined by (2.7). We also use the notation

$$
\int_{[0, s] \times[0, t]} \Psi(u, v) d(u, v) \text { for } J(\Psi)(s, t, \cdot) \text { for }(s, t) \in D .
$$

3. Stochastic differential equations in the plane. The following function spaces are needed in the definition of our stochastic differential equation and its solution.

Let $W$ be the collection of all real valued continuous functions $w$ on $D$. Let

$$
\rho_{W}\left(w_{1}, w_{2}\right)=\sum_{k=1}^{\infty} 2^{-k}\left\{\max _{(s, t)<(k, k)}\left|w_{1}(s, t)-w_{2}(s, t)\right| \wedge 1\right\} \quad \text { for } \quad w_{1}, w_{2} \in W .
$$

Then $\rho_{W}$ is a metric on $W$ and $W$ is a separable complete metric space relative to $\rho_{W}$. A sequence $\left\{w_{n}, n=1,2, \cdots\right\}$ in $W$ converges 
to $w \in W$ in the metric $\rho_{W}$ if and only if $w_{n}$ converges to $w$ uniformly on every bounden subset of $D$.

Let 3 be the collection of cylinder sets $C$ in $W$ of the type $C=\{w \in W ; w(s, t) \in E\}$ for some $(s, t) \in D$ and $E \in \mathfrak{B}(\boldsymbol{R})$. Then $3 \subset W$ and in fact for the $\sigma$-algebra $\mathfrak{B}(W)$ of Borel sets in the metric space $W$ we have

$$
\mathfrak{B}(W)=\sigma(3) .
$$

For $(s, t) \in D$ let $3_{s, t}$ be the collection of the cylinder sets $C$ in $W$ of the type $C=\{w \in W ; w(u, v) \in E\}$ for some $(u, v) \prec(s, t)$ and $E \in \mathfrak{B}(\boldsymbol{R})$. We define

$$
\mathfrak{B}_{s, t}(W)=\sigma\left(\Re_{s, t}\right) .
$$

Let $\partial W$ and $W^{(1)}$ be the collections of all real valued continuous functions on $\partial D$ and $[0, \infty)$ respectively. We define a metric $\rho_{\partial W}$ on $\partial W$ and metric $\rho_{W^{(1)}}$ on $W^{(1)}$ in the same way as we defined $\rho_{W}$ on $W$. Then $\partial W$ and $W^{(1)}$ are separable complete metric spaces with respect to $\rho_{\partial W}$ and $\rho_{W^{(1)}}$ respectively. We write $\mathfrak{B}(\partial W)$ and $\mathfrak{B}\left(W^{(1)}\right)$ for the $\sigma$-algebras of Borel sets in the two metric spaces.

Proposition 3.1. For every transformation $\theta$ of an arbitrary measurable space $(\Omega, \mathfrak{F})$ into the measurable space $(W, \mathfrak{B}(W))$ the following hold:

(1) $\theta$ is $\mathfrak{F} / \mathfrak{B}(W)$ measurable if and only if for every $(s, t) \in D$, $[\theta(\cdot)](s, t)$ is an $\mathfrak{F} / \mathfrak{B}(\boldsymbol{R})$ measurable transformation of $\Omega$ into $\boldsymbol{R}$.

(2) For each $(s, t) \in D, \theta$ is $\mathfrak{F} / \mathfrak{B}_{s, t}(W)$ measurable if and only if for every $(u, v) \prec(s, t),[\theta(\cdot)](u, v)$ is an $\mathfrak{F} / \mathfrak{B}(\boldsymbol{R})$ measurable transformation of $\Omega$ into $\boldsymbol{R}$.

Proof. Since (1) and (2) can be proved in the same way let us prove (2) here. Let $(s, t) \in D$ be fixed.

To prove the necessity of the condition, note that since the transformation of $W$ into $\boldsymbol{R}$ defined by $w \rightarrow w(u, v)$ is $\mathfrak{B}_{s, t}(W) / \mathfrak{B}(\boldsymbol{R})$ measurable for every $(u, v) \prec(s, t)$ assumption of the $\mathfrak{F} / \mathfrak{B}_{s, t}(W)$ measurability of $\theta$ implies the $\widetilde{\Im} / \mathfrak{B}(\boldsymbol{R})$ measurability of $[\theta(\cdot)](u, v)$.

To prove the sufficiency of the condition suppose that for every $(u, v) \prec(s, t),[\theta(\cdot)](u, v)$ is an $\mathfrak{F} / \mathfrak{B}(\boldsymbol{R})$ measurable transformation of $\Omega$ into $R$. Let $C \in \beta_{s, t}$ be given by

$$
C=\{w \in W ; w(u, v) \in E\} \quad \text { where } E \in \mathfrak{B}(\boldsymbol{R}) .
$$

Then from the $\mathfrak{F} / \mathfrak{B}(\boldsymbol{R})$ measurability of $[\theta(\cdot)](u, v)$,

$$
\theta^{-1} C=\{\omega \in \Omega ;[\theta(\omega)](u, v) \in E\} \in \widetilde{\mho} .
$$

From the arbitrariness of $C \in Z_{s, t}$ we have $\theta^{-1}\left(Z_{s, t}\right) \subset \mathfrak{F}$ and therefore 


$$
\theta^{-1}\left(\mathfrak{B}_{s, t}(W)\right)=\theta^{-1}\left(\sigma\left(\Re_{s, t}\right)\right)=\sigma\left(\theta^{-1}\left(\Re_{s, t}\right)\right) \subset \mathfrak{\mho}
$$

i.e., $\theta$ is $\widetilde{\mho} / \mathfrak{R}_{s, t}(W)$ measurable.

Definition 3.2. We write $\boldsymbol{M}(D \times W)$ for the collection of all real valued functions $\alpha$ on $D \times W$ satisfying the following measurability conditions:

$1^{\circ} \quad \alpha$ is a $\sigma(\mathfrak{B}(D) \times \mathfrak{B}(W)) / \mathfrak{B}(\boldsymbol{R})$ measurable transformation of $D \times W$ into $\boldsymbol{R}$.

$2^{\circ}$ For every $(s, t) \in D, \alpha(s, t, \cdot)$ is a $\mathfrak{B}_{s, t}(W) / \mathfrak{B}(\boldsymbol{R})$ measurable transformation of $W$ into $\boldsymbol{R}$.

Definition 3.3. Let $\alpha, \beta \in \boldsymbol{M}(D \times W)$. By a solution of the stochastic differential equation

$$
d X(s, t)=\alpha(s, t, X) d B(s, t)+\beta(s, t, X) d(s, t)
$$

we mean a system of two 2-parameter stochastic processes $(X, B)$ on an equipped probability space $\left(\Omega, \widetilde{F}, P ; \widetilde{\mho}_{s, t}\right)$ satisfying the following conditions:

$1^{\circ} \quad B$ is an $\widetilde{\mho}_{s, t}$ Brownian motion with $\partial B=0$.

$2^{\circ} \quad X$ is an $\widetilde{\mho}_{s, t}$ adapted process whose sample functions are all continuous on $D$.

$3^{\circ}$ If we set

$$
\begin{aligned}
& \Phi(s, t, \omega)=\alpha(s, t, X(\cdot, \cdot \omega)) \text { for } \quad(s, t, \omega) \in D \times \Omega \\
& \Psi(s, t, \omega)=\beta(s, t, X(\cdot, \cdot, \omega)) \text { for } \quad(s, t, \omega) \in D \times \Omega
\end{aligned}
$$

then $\Phi$ and $\Psi$ are in the classes $\mathfrak{R}_{2}\left(\widetilde{\mho}_{s, t}\right)$ and $\mathfrak{R}_{1}\left(\mathfrak{\mho}_{s, t}\right)$ respectively.

$4^{\circ}$ With probability 1

$$
\begin{aligned}
& X(s, t)-X(s, 0)-X(0, t)+X(0,0) \\
& =\int_{[0, s] \times[0, t]} \alpha(u, v, X) d B(u, v)+\int_{[0, s] \times[0, t]} \beta(u, v, X) d(u, v) \\
& \text { for all }(s, t) \in D .
\end{aligned}
$$

REMARK 3.4. The condition $2^{\circ}$ of Definition 3.3 is equivalent to the condition that $X$ is an $\mathfrak{F} / \mathfrak{B}(W)$ measurable transformation of $\Omega$ into $W$ and furthermore for every $(s, t) \in D, X$ is an $\mathfrak{\mho}_{s, t} / \mathfrak{B}_{s, t}(W)$ measurable transformation of $\Omega$ into $W$.

REMark 3.5. $\Phi$ and $\Psi$ as defined in $3^{\circ}$ of Definition 3.3 are measurable and $\widetilde{F}_{s, t}$ adapted processes on $\left(\Omega, \mathfrak{F}, P ; \widetilde{F}_{s, t}\right)$. The assumption in $3^{\circ}$ that $\Phi$ and $\Psi$ are in the classes $\mathfrak{R}_{2}\left(\widetilde{\mho}_{s, t}\right)$ and $\mathfrak{L}_{1}\left(\widetilde{\mho}_{s, t}\right)$ respectively implies according to Proposition 2.5 that they have equivalent processes on $\left(\Omega, \mathfrak{F}, P ; \mathfrak{F}_{s, t}\right)$ which are progressively measurable. 
Therefore we shall always assume that $\Phi$ and $\Psi$ themselves are progressively measurable.

Definition 3.6. A solution $(X, B)$ of the stochastic differential equation (3.1) on an equipped probability space $\left(\Omega, \widetilde{F}, P ; \mathfrak{F}_{s, t}\right)$ is called a strong solution if there exists a transformation $F$ of $\partial W \times W$ into $W$ satisfying the following conditions:

$1^{\circ} \quad F$ is $\sigma(\mathfrak{B}(\partial W) \times \mathfrak{B}(W)) / \mathfrak{B}(W)$ measurable.

$2^{\circ} \quad$ For each $z \in \partial W, F[z, \cdot]$ is a $\mathfrak{B}_{s, t}(W) / \mathfrak{B}_{s, t}(W)$ measurable transformation of $W$ into $W$ for every $(s, t) \in D$.

$3^{\circ}$ With the boundary stochastic process $\partial X$ of $X$ we have

$$
X(\cdot, \cdot, \omega)=F[\partial X(\cdot, \cdot, \omega), B(\cdot, \cdot, \omega)] \text { for a.e. } \omega \in \Omega \text {. }
$$

DEFINITION 3.7. We say that the solution of the stochastic differential equation (3.1) is pathwise unique if whenever there are two solutions $(X, B)$ and $\left(X^{\prime}, B\right)$ with the same $B$ on the same equipped probability space $\left(\Omega, \mathfrak{F}, P ; \mathfrak{F}_{s, t}\right)$ such that $\partial X=\partial X^{\prime}$, then $X=X^{\prime}$.

As sufficient conditions for the pathwise uniqueness of the solution and for the existence of a strong solution of the stochastic differential equation (3.1) we have the following Lipschitz condition and growth condition on the coefficients $\alpha, \beta$ in (3.1): There exists a Borel measure $\lambda$ on $D$ which is finite for every compact subset of $D$ and satisfies the condition that for every $T>0$ there exists $L_{T}>0$ such that

$$
\begin{aligned}
& \begin{aligned}
\mid \alpha(s, t, w)- & \left.\alpha\left(s, t, w^{\prime}\right)\right|^{2}+\left|\beta(s, t, w)-\beta\left(s, t, w^{\prime}\right)\right|^{2} \\
\leqq & L_{T}\left\{\int_{[0, s] \times[0, t]}\left|w(u, v)-w^{\prime}(u, v)\right|^{2} \lambda(d(u, v))\right. \\
& \left.\quad+\left|w(s, t)-w^{\prime}(s, t)\right|^{2}\right\}, \\
|\alpha(s, t, w)|^{2} & +|\beta(s, t, w)|^{2} \\
\leqq & L_{T}\left\{\int_{[0, s] \times[0, t]}|w(u, v)|^{2} \lambda(d(u, v))+|w(s, t)|^{2}+1\right\}
\end{aligned}
\end{aligned}
$$

for all $(s, t) \prec(T, T)$ and $w, w^{\prime} \in W$. We shall show by the method of successive approximation that (3.3) ensures the pathwise uniqueness of the solution and that (3.3) and (3.4) together ensure the existence of a strong solution for (3.1).

THEOREM 3.8. If the coefficients $\alpha$ and $\beta$ in the stochastic differential equation (3.1) in Definition 3.3 satisfy the Lipschitz condition (3.3) then its solution is pathwise unique. 
Proof. Let $(X, B)$ and $\left(X^{\prime}, B\right)$ be two solutions of (3.1) on the same equipped probability space $\left(\Omega, \mathfrak{F}, P ; \mathfrak{\mho}_{s, t}\right)$ with $\partial X=\partial X^{\prime}$. We proceed to show that $X=X^{\prime}$. Since the sample functions of $X$ and $X^{\prime}$ are all continuous on $D$, it suffices to show that for each $(s, t) \in D$ we have $X(s, t)=X^{\prime}(s, t)$, i.e., $X(s, t, \omega)=X^{\prime}(s, t, \omega)$ for a.e. $\omega \in \Omega$. We shall show this by showing

$$
P\left\{\left|X(s, t)-X^{\prime}(s, t)\right|>0\right\}=0 \quad \text { for every } \quad(s, t) \in D .
$$

Now since both $X$ and $X^{\prime}$ satisfy (3.2) and since $\partial X=\partial X^{\prime}$, we have with probability 1

$$
\begin{aligned}
& X(s, t)-X^{\prime}(s, t)= \int_{[0, s] \times[0, t]}\left\{\alpha(u, v, X)-\alpha\left(u, v, X^{\prime}\right)\right\} d B(u, v) \\
&+\int_{[0, s] \times[0, t]}\left\{\beta(u, v, X)-\beta\left(u, v, X^{\prime}\right)\right\} d(u, v) \\
& \text { for all }(s, t) \in D .
\end{aligned}
$$

For each positive integer $N$ and $(s, t) \in D$ let

$$
A_{s, t}^{N}=\left\{\omega \in \Omega ; \sup _{\langle u, v\rangle<\langle s, t\rangle}\left(|X(u, v, \omega)|^{2}+\left|X^{\prime}(u, v, \omega)\right|^{2}\right) \leqq N\right\} .
$$

Since $|X|$ and $\left|X^{\prime}\right|$ are $\widetilde{\mho}_{s, t}$ adapted processes on $\left(\Omega, \mathfrak{F}, P ; \mathfrak{F}_{s, t}\right)$, $|X(u, v, \cdot)|$ and $\left|X^{\prime}(u, v, \cdot)\right|$ are $\mathfrak{\mho}_{s, t}$ measurable random variables for every $(u, v) \prec(s, t)$. This implies that $A_{s, t}^{N} \in \mathfrak{\mho}_{s, t}$ for each $(s, t) \in D$. Let us write $\chi(\cdot, A)$ for the characteristic function of a subset $A$ of $\Omega$ and consider the $\{0,1\}$ valued 2-parameter stochastic process $I^{N}$ on $\left(\Omega, \mathfrak{\mho}, P ; \mathfrak{\mho}_{s, t}\right)$ defined by

$$
I^{N}(s, t, \omega)=\chi\left(\omega, A_{s, t}^{N}\right) \quad \text { for } \quad(s, t, \omega) \in D \times \Omega .
$$

Since $A_{s, t}^{N} \in \mathfrak{F}_{s, t}$ for every $(s, t) \in D, I^{N}$ is an $\widetilde{\mho}_{s, t}$ adapted process. Also since the sample functions of $|X|$ and $\left|X^{\prime}\right|$ are all continuous and since for every $\omega \in \Omega$ the function $f_{\omega}$ defined by

$$
f_{\omega}(s, t)=\sup _{(u, v)<(s, t)}\left(|X(u, v, \omega)|^{2}+\left|X^{\prime}(u, v, \omega)\right|^{2}\right) \quad \text { for } \quad(s, t) \in D
$$

is an increasing function on $D$ in the sense of the partial order $\prec$, each sample function of $I^{N}$ is left continuous in the sense that

$$
\lim _{\substack{(u, v) \rightarrow(s, t) \\(u, v)<(s, t)}} I^{N}(u, v, \omega)=I^{N}(s, t, \omega) \text { for }(s, t) \in D .
$$

Therefore by Proposition $2.3, I^{N}$ is a progressively measurable process on $\left(\Omega, \mathfrak{\mho}, P ; \widetilde{\mho}_{s, t}\right)$. Now

$$
\left\{\chi\left(\omega, A_{s t}^{N}\right)\right\}^{2}=\chi\left(\omega, A_{s, t}^{N}\right)
$$

and 


$$
(u, v) \prec(s, t) \Rightarrow A_{u, v}^{N} \supset A_{s, t}^{N} \Rightarrow \chi\left(\omega, A_{u, v}^{N}\right) \chi\left(\omega, A_{s, t}^{N}\right)=\chi\left(\omega, A_{s, t}^{N}\right)
$$

and consequently

$$
\begin{gathered}
\left\{I^{N}(s, t, \omega)\right\}^{2}=I^{N}(s, t, \omega), \\
(u, v) \prec(s, t) \Rightarrow I^{N}(u, v, \omega) \geqq I^{N}(s, t, \omega), \\
(u, v) \prec(s, t) \Rightarrow I^{N}(u, v, \omega) I^{N}(s, t, \omega)=I^{N}(s, t, \omega) .
\end{gathered}
$$

By (2), (5) and (6) we have with probability 1

$$
\begin{aligned}
& I^{N}(s, t)\left\{X(s, t)-X^{\prime}(s, t)\right\}^{2} \\
& \leqq 2 I^{N}(s, t)\left[\{I(\Phi)(s, t)\}^{2}+\{J(\Psi)(s, t)\}^{2}\right] \text { for all }(s, t) \in D
\end{aligned}
$$

where $\Phi$ and $\Psi$ are defined by

$$
\begin{aligned}
& \Phi(s, t, \omega)=I^{N}(s, t, \omega)\left[\alpha(s, t, X(\cdot, \cdot, \omega))-\alpha\left(s, t, X^{\prime}(\cdot, \cdot, \omega)\right)\right], \\
& \Psi(s, t, \omega)=I^{N}(s, t, \omega)\left[\beta(s, t, X(\cdot, \cdot, \omega))-\beta\left(s, t, X^{\prime}(\cdot, \cdot, \omega)\right)\right]
\end{aligned}
$$

for $(s, t, \omega) \in D \times \Omega$ and $I(\Phi)$ and $J(\Psi)$ are stochastic integrals of $\Phi$ and $\Psi$ with respect to the $\widetilde{\mho}_{s, t}$ Brownian motion and the Lebesgue measure respectively as in (2.4) and (2.8) of $\S 2$. Note that by $3^{\circ}$ of Definition 3.3, $\Phi$ and $\Psi$ are 2-parameter stochastic processes on $\left(\Omega, \mathfrak{F}, P ; \widetilde{\mho}_{s, t}\right)$ of the class $\mathfrak{R}_{2}\left(\widetilde{\mho}_{s, t}\right)$ and $\mathfrak{L}_{1}\left(\widetilde{\mho}_{s, t}\right)$ respectively. From the last inequality we have

$$
\begin{aligned}
E\left[I^{N}(s, t)\left\{X(s, t)-X^{\prime}(s, t)\right\}^{2}\right] & \\
& \leqq 2 \boldsymbol{E}\left[|I(\Phi)(s, t)|^{2}\right]+2 \boldsymbol{E}\left[|J(\Psi)(s, t)|^{2}\right] \quad \text { for } \quad(s, t) \in D .
\end{aligned}
$$

Let us show that for every $(s, t) \in D, \Phi$ and $\Psi$ are bounded on $[0, s] \times[0, t] \times \Omega$. Consider $\Phi$ for instance. Let $T>0$ be so large that $(s, t) \prec(T, T)$. By applying (5) and the Lipschitz condition (3.3) to (8) we have for every $\omega \in \Omega$

$$
\begin{aligned}
|\Phi(u, v, \omega)|^{2} & \\
\leqq & I^{N}(u, v, \omega) L_{T}\left\{\int_{[0, u] \times[0, v]}\left|X\left(u^{\prime}, v^{\prime}, \omega\right)-X^{\prime}\left(u^{\prime}, v^{\prime}, \omega\right)\right|^{2} \lambda\left(d\left(u^{\prime}, v^{\prime}\right)\right)\right. \\
& \left.+\left|X(u, v, \omega)-X^{\prime}(u, v, \omega)\right|^{2}\right\} \text { for }(u, v) \prec(s, t) .
\end{aligned}
$$

Taking $I^{N}(u, v, \omega)$ under the integral sign for the first term on the right side and then recalling (4) and (3) we have for every $\omega \in \Omega$

$$
|\Phi(u, v, \omega)|^{2} \leqq L_{T}\{2 N \lambda([0, s] \times[0, t])+2 N\} \text { for }(u, v) \prec(s, t) .
$$

This proves the boundedness of $\Phi$ on $[0, s] \times[0, t] \times \Omega$. The same estimate holds for $\Psi$. Also this implies in particular that $\Psi$ belongs not only in the class $\mathfrak{R}_{1}\left(\mathfrak{\mho}_{s, t}\right)$ but also in the class $\mathfrak{R}_{2}\left(\mathfrak{\mho}_{s, t}\right)$. 
Returning to the stochastic integral $I(\Phi)$ we have by $(2.6)$ in $\S 2$ and the Fubini theorem

$$
\boldsymbol{E}\left[|I(\Phi)(s, t)|^{2}\right]=\int_{[0, s] \times[0, t]} \boldsymbol{E}\left[|\Phi(u, v)|^{2}\right] m_{L}(d(u, v)) .
$$

For the stochastic integral $J(\Psi)$ we have by the Schwarz Inequality and the Fubini theorem

$$
\boldsymbol{E}\left[|J(\Psi)(s, t)|^{2}\right] \leqq s t \int_{[0, s] \times[0, t]} \boldsymbol{E}\left[|\Psi(u, v, \cdot)|^{2}\right] m_{L}(d(u, v)) .
$$

Using (11), (12) in (10), applying (3.3) and recalling that $I^{N}(u, v) \leqq$ $I^{N}\left(u^{\prime}, v^{\prime}\right)$ for $\left(u^{\prime}, v^{\prime}\right) \prec(u, v)$ by $(6)$ we obtain

$$
\begin{aligned}
& E\left[I^{N}(s, t)\left\{X(s, t)-X^{\prime}(s, t)\right\}^{2}\right] \\
& \leqq 2(1+s t) \int_{[0, s] \times[0, t]} E\left[|\Phi(u, v)|^{2}+|\Psi(u, v)|^{2}\right] m_{L}(d(u, v)) \\
& \leqq 2(1+s t) L_{T} \int_{[0, s] \times[0, t]} \boldsymbol{E}\left[\int_{[0, u] \times[0, v]} I^{N}\left(u^{\prime}, v^{\prime}\right)\right. \\
& \times\left\{X\left(u^{\prime}, v^{\prime}\right)-X^{\prime}\left(u^{\prime}, v^{\prime}\right)\right\} \lambda\left(d\left(u^{\prime}, v^{\prime}\right)\right) \\
& \left.+I^{N}(u, v)\left\{X(u, v)-X^{\prime}(u, v)\right\}^{2}\right] m_{L}(d(u, v)) \\
& \text { for }(s, t) \prec(T, T) \text {. }
\end{aligned}
$$

Let

$$
c(s, t)=\sup _{(u, v)<(s, t)} E\left[I^{N}(u, v)\left\{X(u, v)-X^{\prime}(u, v)\right\}^{2}\right] \quad \text { for } \quad(s, t) \in D .
$$

Using (14) in (13) we obtain

$$
\begin{aligned}
& E\left[I^{N}(s, t)\left\{X(s, t)-X^{\prime}(s, t)\right\}^{2}\right] \\
& \leqq 2\left(1+T^{2}\right) L_{T}\{\lambda([0, T] \times[0, T])+1\} \int_{[0, s] \times[0, t]} c(u, v) m_{L}(d(u, v)) \\
& \text { for } \quad(s, t) \prec(T, T) .
\end{aligned}
$$

Since $c$ is nonnegative, the last inequality implies

$$
\begin{aligned}
& \boldsymbol{E}\left[I^{N}(u, v)\left\{X(u, v)-X^{\prime}(u, v)\right\}^{2}\right] \\
& \leqq 2\left(1+T^{2}\right) L_{T}\{\lambda([0, T] \times[0, T])+1\} \int_{[0, s] \times[0, t]} c\left(u^{\prime}, v^{\prime}\right) m_{L}\left(d\left(u^{\prime}, v^{\prime}\right)\right) \\
& \text { for }(u, v) \prec(s, t) \prec(T, T) .
\end{aligned}
$$

Taking the supremum over $(u, v) \prec(s, t)$ on the left side we have by (14)

$$
\begin{aligned}
c(s, t) \leqq & 2\left(1+T^{2}\right) L_{T}\{\lambda([0, T] \times[0, T])+1\} \\
& \times \int_{[0, s] \times[0, t]} c(u, v) m_{L}(d(u, v)) \text { for } \quad(s, t) \prec(T, T) .
\end{aligned}
$$


Now for every $T>0$, the Borel measurable function $c$ on $D$ is bounded on $[0, T] \times[0, T]$ since

$$
c(s, t) \leqq c(T, T)<\infty \quad \text { for } \quad(s, t) \prec(T, T)
$$

by (14), (10) and the boundedness of $\Phi$ and $\Psi$ on $[0, T] \times[0, T] \times \Omega$. For brevily let us write

$$
a=2\left(1+T^{2}\right) L_{T}\{\lambda([0, T] \times[0, T])+1\} .
$$

Then by iterating (15) $n$ times we have

$$
\begin{aligned}
c(s, t) \leqq & a^{n+1} \int_{[0, s] \times[0, t]} \int_{\left[0, u_{1}\right] \times\left[0, v_{1}\right]} \cdots \int_{\left[0, u_{n}\right] \times\left[0, v_{n}\right]} c\left(u_{n+1}, v_{n+1}\right) \\
& \times m_{L}\left(d\left(u_{n+1}, v_{n+1}\right)\right) \cdots m_{L}\left(d\left(u_{2}, v_{2}\right)\right) m_{L}\left(d\left(u_{1}, v_{1}\right)\right) \\
\leqq & a^{n+1} c(T, T) \frac{(s t)^{n+1}}{\{(n+1) !\}^{2}} \quad \text { for } \quad(s, t) \prec(T, T) .
\end{aligned}
$$

Since this holds for every positive integer $n$ and since $T>0$ is arbitrary, we have

$$
c(s, t)=0 \quad \text { for } \quad(s, t) \in D .
$$

Using this in (14) and recalling (4) we have

$$
\boldsymbol{E}\left[\chi\left(\cdot, A_{s, t}^{N}\right)\left\{X(s, t)-X^{\prime}(s, t)\right\}^{2}\right]=0 \quad \text { for } \quad(s, t) \in D .
$$

Since the second factor in the expectation is nonnegative, the last equality implies

$$
\left\{\omega \in \Omega ;\left|X(s, t, \omega)-X^{\prime}(s, t, \omega)\right|>0\right\} \subset\left(A_{s t}^{N}\right)^{c} \cup A_{s t}^{*} \quad \text { for } \quad(s, t) \in D
$$

where $A_{s t}^{*}$ is a null set in $(\Omega, \mathfrak{F}, P)$. Thus

$$
P\left\{\left|X(s, t)-X^{\prime}(s, t)\right|>0\right\} \leqq P\left(\left(A_{s, t}^{N}\right)^{c}\right) \text { for } \quad(s, t) \in D .
$$

Since $\left\{A_{s, t}^{N}, N=1,2, \cdots\right\}$ is a monotone increasing sequence of sets, $\left\{P\left(\left(A_{s, t}^{N}\right)^{c}\right), N=1,2, \cdots\right\}$ is a monotone decreasing sequence of nonnegative numbers. To show that it actually converges to 0 , assume the contrary. Then there exists $\varepsilon>0$ such that

$$
P\left(\bigcap_{N=1}^{\infty}\left(A_{s, t}^{N}\right)^{c}\right)=\lim _{N \rightarrow \infty} P\left(\left(A_{s, t}^{N}\right)^{c}\right) \geqq \varepsilon .
$$

In other words

$$
P\left\{\omega \in \Omega ; \sup _{(u, v)<(s, t)}\left(|X(u, v, \omega)|^{2}+\left|X^{\prime}(u, v, \omega)\right|^{2}\right)>N \text { for all } N\right\} \geqq \varepsilon,
$$
i.e.,

$$
P\left\{\omega \in \Omega ; \sup _{(u, v)<(s, t)}\left(|X(u, v, \omega)|^{2}+\left|X^{\prime}(u, v, \omega)\right|^{2}\right)=\infty\right\} \geqq \varepsilon
$$


This contradicts the fact that the sample functions of $X$ and $X^{\prime}$ are all continuous on $D$ and consequently the supremum in the above expression is finite for every $\omega \in \Omega$. Therefore

$$
\lim _{N \rightarrow \infty} P\left(\left(A_{s, t}^{N}\right)^{c}\right)=0 \text {. }
$$

Letting $N \rightarrow \infty$ on the right side of (16) and applying (17) we have (1).

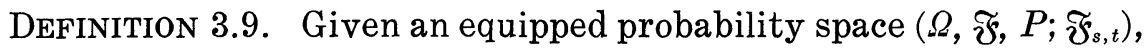
let $\boldsymbol{L}_{2}^{c}\left(\mathfrak{\mho}_{s, t}\right)$ be the collection of 2-parameter stochastic processes $X$ on $\left(\Omega, \widetilde{\Im}, P ; \widetilde{\mho}_{s, t}\right)$ which satisfy the following conditions:

$1^{\circ} \quad X$ is $\Im_{s, t}$ adapted.

$2^{\circ}$ Every sample function of $X$ is continuous on $D$.

$3^{\circ}$ For every $T>0$

$$
\gamma(T ; X) \equiv \sup _{(s, t)<(T, T)} \boldsymbol{E}\left[|X(s, t)|^{2}\right]<\infty .
$$

Let $\boldsymbol{L}_{2}^{c}\left(\mathfrak{\mho}_{s, t} \mid \partial D\right)$ be the collection of boundary stochastic processes $Z$ on $\left(\Omega, \widetilde{\mho}, P ; \widetilde{\mho}_{s, t}\right)$ which satisfy the following conditions:

$4^{\circ} Z$ is $\widetilde{F}_{s, t}$ adapted.

$5^{\circ}$ Every sample function of $Z$ is continuous on $\partial D$.

$6^{\circ} \quad$ For every $T>0$

$$
\gamma(T ; Z) \equiv \sup _{\substack{(s, t)<(T, T) \\(s, t) \in \partial D}} \boldsymbol{E}\left[|Z(s, t)|^{2}\right]<\infty .
$$

Note that if $X$ is in $\boldsymbol{L}_{2}^{c}\left(\mathfrak{\mho}_{s, t}\right)$, then $X$ is of the class $\mathfrak{\Omega}_{2}\left(\mathfrak{\mho}_{s, t}\right)$.

LEMMA 3.10. Let $\left(\Omega, \mathfrak{\mho}, P ; \widetilde{\mho}_{s, t}\right)$ be an equipped probability space on which an $\mathfrak{\mho}_{s, t}$ Brownian motion $B$ with $\partial B=0$ exists. Suppose that the coefficients $\alpha$ and $\beta$ in the stochastic differential equation (3.1) in Definition 3.3 satisfy the growth condition (3.4). Let $Z \in$ $\boldsymbol{L}_{2}^{c}\left(\mathfrak{F}_{s, t} \mid \partial D\right)$ be fixed .

For every $X \in \boldsymbol{L}_{2}^{c}\left(\mathfrak{\mho}_{s, t}\right)$ define a 2-parameter stochastic process $\tau X$ on $\left(\Omega, \mathfrak{F}, P ; \mathfrak{\Im}_{s, t}\right)$ by

$$
\begin{aligned}
(\tau X)(s, t)= & -Z(0,0)+Z(s, 0)+Z(0, t) \\
& +\int_{[0, s] \times[0, t]} \alpha(u, v, X) d B(u, v) \\
& +\int_{[0, s] \times[0, t]} \beta(u, v, X) d(u, v) \text { for } \quad(s, t) \in D .
\end{aligned}
$$

Then

$$
\tau X \in \boldsymbol{L}_{2}^{c}\left(\Im_{s, t}\right)
$$


With the transformation $\tau$ of $\boldsymbol{L}_{2}^{c}\left(\mathfrak{\mho}_{s, t}\right)$ into itself as given above, define a sequence $\left\{X^{(i)}, i=0,1,2, \cdots\right\}$ in $L_{2}^{c}\left(\mathfrak{F}_{s, t}\right)$ by

$$
\left\{\begin{array}{l}
X^{(0)}(s, t)=-Z(0,0)+Z(s, 0)+Z(0, t) \text { for }(s, t) \in D, \\
X^{(i)}(s, t)=\left(\tau X^{(i-1)}\right)(s, t) \text { for }(s, t) \in D \text { and } i=1,2, \cdots
\end{array}\right.
$$

Then for every $T>0$ there exists $M_{T}>0$ such that

$$
\sup _{(s, t)<(T, T)} \boldsymbol{E}\left[\left|X^{(i)}(s, t)\right|^{2}\right] \leqq M_{T} \quad \text { for } \quad i=0,1,2, \cdots .
$$

Proof. Let $X \in \boldsymbol{L}_{2}^{c}\left(\mathfrak{\mho}_{s, t}\right)$. As we saw in Remark 3.5, the fact that $\alpha, \beta \in M(D \times W)$ and $X$ is an $\widetilde{\mho}_{s, t}$ adapted process whose sample functions are all continuous on $D$ implies that the two 2-parameter stochastic process $\Phi$ and $\Psi$ on $\left(\Omega, \mathfrak{F}, P ; \widetilde{\mho}_{s, t}\right)$ defined by

$$
\left\{\begin{array}{l}
\Phi(s, t, \omega)=\alpha(s, t, X(\cdot, \cdot \omega)) \\
\Psi(s, t, \omega)=\beta(s, t, X(\cdot, \cdot, \omega))
\end{array}\right.
$$

are $\widetilde{F}_{s, t}$ adapted and measurable processes. Furthermore the assumption that $\alpha$ and $\beta$ satisfy (3.4) implies that $\Phi$ and $\Psi$ are both of the class $\mathfrak{Q}_{2}\left(\mathfrak{F}_{s, t}\right)$. In fact for every $T>0$ we have by (3.4)

$$
\begin{aligned}
E\left[\int_{[0, T] \times[0, T]}\right. & \left.\left\{|\Phi(s, t)|^{2}+|\Psi(s, t)|^{2}\right\} m_{L}(d(s, t))\right] \\
\leqq & E\left[\int _ { [ 0 , T ] \times [ 0 , T ] } L _ { T } \left\{\int_{[0, s] \times[0, t]}|X(u, v)|^{2} \lambda(d(u, v))\right.\right. \\
& \left.\left.\quad+|X(s, t)|^{2}+1\right\} m_{L}(d(s, t))\right] \\
\leqq & L_{T}[\gamma(T ; X)\{\lambda([0, T] \times[0, T])+1\}+1] T^{2}<\infty .
\end{aligned}
$$

Now that $\Phi$ and $\Psi$ are of the class $\mathcal{R}_{2}\left(\widetilde{\mho}_{s, t}\right)$ the two stochastic integrals in (1) exist and $\tau X$ is defined. Clearly $\tau X$ is an $\widetilde{\mho}_{s, t}$ adapted process whose sample functions are all continuous on $D$.

To show that $\tau X$ is in $\boldsymbol{L}_{2}^{c}\left(\widetilde{\mho}_{s, t}\right)$ it remains to verify that for every $T>0$

$$
\sup _{(s, t)<(T, T)} \boldsymbol{E}\left[|(\tau X)(s, t)|^{2}\right]<\infty .
$$

This can be shown by the same kind of estimates used in (6). However we shall obtain a more precise estimate than (7) which is needed in proving (4) and at the same time implies (7). First of all, for any $X \in \boldsymbol{L}_{2}^{c}\left(\mathfrak{F}_{s, t}\right)$ there exists a nonnegative Borel measurable function $A(s, t ; X)$ for $(s, t) \prec(T, T)$ which is increasing in the sense of " $\prec$ " in $D$ and satisfies the condition

$$
\sup _{(u, v)<(s, t)} E\left[|X(u, v)|^{2}\right]+1 \leqq A(s, t ; X) \quad \text { for } \quad(s, t) \prec(T, T) .
$$


Indeed the fact that $X$ is in $\boldsymbol{L}_{2}^{c}\left(\widetilde{\mho}_{s, t}\right)$ implies that

$$
\sup _{(u, v)<(s, t)} \boldsymbol{E}\left[|X(u, v)|^{2}\right] \leqq \gamma(s \vee t ; X)
$$

so that if we define

$$
A(s, t ; X)=\gamma(s \vee t ; X)+1 \text { for }(s, t) \prec(T, T),
$$

then $A(\cdot, \cdot ; X)$ is increasing in the sense of " $\prec$ " in $D$ and (8) holds. We show next that for any increasing function $A(\cdot, \cdot ; X)$ on $[0, T] \times[0, T]$ which satisfies (8) we have

$$
\begin{gathered}
E\left[|(\tau X)(s, t)|^{2}\right] \leqq 5\left\{3 \gamma(T ; Z)+B_{T} \int_{[0, s] \times[0, t]} A(u, v ; X) m_{L}(d(u, v))\right\} \\
\text { for } \quad(s, t) \prec(T, T)
\end{gathered}
$$

where

$$
B_{T}=\left(1+T^{2}\right) L_{T}\{\lambda([0, T] \times[0, T])+1\} .
$$

Clearly (9) and (10) imply (7). To prove (9) note that from (1) and (5)

$$
\begin{aligned}
|(\tau X)(s, t)|^{2} \leqq & 5\left\{|Z(0,0)|^{2}+|Z(s, 0)|^{2}+|Z(0, t)|^{2}+|I(\Phi)(s, t)|^{2}\right. \\
& \left.+|J(\Psi)(s, t)|^{2}\right\} \quad \text { for } \quad(s, t) \prec(T, T)
\end{aligned}
$$

and therefore by (11) and (12) in the proof of Theorem 3.8 and then by (3.4) and (8) we have

$$
\begin{aligned}
& \boldsymbol{E}\left[|(\tau X)(s, t)|^{2}\right] \\
& \leqq 5\left\{3 \gamma(T ; Z)+\int_{[0, s] \times[0, t]} \boldsymbol{E}\left[|\Phi(u, v)|^{2}\right] m_{L}(d(u, v))\right. \\
&\left.+s t \int_{[0, s] \times[0, t]} \boldsymbol{E}\left[|\Psi(u, v)|^{2}\right] m_{L}(d(u, v))\right\} \\
& \leqq 5\left\{3 \gamma(T ; Z)+(1+s t) \int_{[0, s] \times[0, t]} \boldsymbol{E}\left[|\alpha(u, v, X)|^{2}\right.\right. \\
&\left.\left.+|\beta(u, v, X)|^{2}\right] m_{L}(d(u, v))\right\} \\
& \leqq 5\left\{3 \gamma(T ; Z)+\left(1+T^{2}\right) L_{T} \int_{[0, s] \times[0, t]} \boldsymbol{E}\left[\int_{[0, u] \times[0, v]}\left|X\left(u^{\prime}, v^{\prime}\right)\right|^{2}\right.\right. \\
&\left.\left.\times \lambda\left(d\left(u^{\prime}, v^{\prime}\right)\right)+|X(u, v)|^{2}+1\right] m_{L}(d(u, v))\right\} \\
& \leqq 5\left\{3 \gamma(T ; Z)+\left(1+T^{2}\right) L_{T}[\lambda([0, T] \times[0, T])+1]\right. \\
&\left.\times \int_{[0, s] \times[0, t]} A(u, v ; X) m_{L}(d(u, v))\right\},
\end{aligned}
$$

which proves (9) in view of (10).

We now turn to the proof of (4) under the definition of 
$\left\{X^{(i)}, i=0,1,2, \cdots\right\}$ by (3). Clearly $X^{(0)}$ is an $\mathfrak{\mho}_{s, t}$ adapted process on $\left(\Omega, \mathfrak{F}, P ; \mathfrak{\mho}_{s, t}\right)$ whose sample functions are all continuous on $D$ and

$$
\begin{aligned}
\gamma\left(T ; X^{(0)}\right) & =\sup _{(s, t)<(T, T)} \boldsymbol{E}\left[\left|X^{(0)}(s, t)\right|^{2}\right] \\
& \leqq 3 \sup _{0 \leqq s, t \leqq T}\left\{|Z(0,0)|^{2}+|Z(s, 0)|^{2}+|Z(0, t)|^{2}\right\} \\
& \leqq 3 \gamma(T ; Z)<\infty .
\end{aligned}
$$

Thus $X^{(0)} \in \boldsymbol{L}_{2}^{c}\left(\mathfrak{\mho}_{s, t}\right)$. Then by (2) we have $X^{(i)} \in \boldsymbol{L}_{2}^{c}\left(\mathfrak{\mho}_{s, t}\right)$ for $i=1,2, \cdots$ also.

To prove (4) let us write for brevity

$$
c=3 \gamma(T: Z)
$$

and show by induction on $i$ that

$$
\begin{aligned}
E\left[\left|X^{(i)}(s, t)\right|^{2}\right] \leqq & 5\left\{c \sum_{k=0}^{i-1}\left(5 B_{T} s t\right)^{k}(k !)^{-2}+5^{i-1}(c+1)\left(B_{T} s t\right)^{i}(i !)^{-2}\right. \\
& \left.+\sum_{k=1}^{i-1} 5^{k-1}\left(B_{T} s t\right)^{k}(k !)^{-2}\right\} \\
& \quad \text { for } \quad(s, t) \prec(T, T) \text { and } i=1,2, \cdots .
\end{aligned}
$$

To show that (12) holds for $i=1$ let

$$
A\left(s, t ; X^{(0)}\right)=3 c+1 \text { for }(s, t) \prec(T, T) .
$$

Then $A$ is trivially a nonnegative Borel measurable function which is increasing in the sense of " $\prec$ " in $D$. Also by (11), $A$ satisfies (8) with $X^{(0)}$ in the place of $X$ so that by (9)

$$
\boldsymbol{E}\left[\left|X^{(1)}(s, t)\right|^{2}\right] \leqq 5\left\{c+(c+1) B_{T} s t\right\},
$$

which is (12) for $i=1$. Next assume that (12) holds for some positive integer $i$. Define

$$
\begin{aligned}
A\left(s, t ; X^{(i)}\right)= & 5\left\{c \sum_{k=0}^{i-1}\left(5 B_{T} s t\right)^{k}(k !)^{-2}+5^{i-1}(c+1)\left(B_{T} s t\right)^{i}(i !)^{-2}\right. \\
& \left.+\sum_{k=1}^{i-1} 5^{k-1}\left(B_{T} s t\right)^{k}(k !)^{-2}\right\}+1 \text { for } \quad(s, t) \prec(T, T) .
\end{aligned}
$$

Then $A$ is a nonnegative Borel measurable function which is increasing in the sense of " $\prec$ " in $D$ and satisfies (8) with $X^{(i)}$ in the place of $X$. Thus by (9)

$$
\begin{aligned}
\boldsymbol{E}\left[\left|X^{(i+1)}(s, t)\right|^{2}\right] \leqq & 5\left\{c+B_{T} \int_{[0, s] \times[0, t]} A\left(u, v ; X^{(i)}\right) m_{L}(d(u, v))\right\} \\
= & 5\left\{c \sum_{k=0}^{i}\left(5 B_{T} s t\right)^{k}(k !)^{-2}+5^{i}(c+1)\left(B_{T} s t\right)^{i+1}[(i+1) !]^{-2}\right. \\
& \left.+\sum_{k=1}^{i} 5^{k-1}\left(B_{T} s t\right)^{k}(k !)^{-2}\right\},
\end{aligned}
$$


which is (12) for $i+1$. Thus by induction (12) holds for $i=1,2, \cdots$. From (12) we obtain the simpler estimate

$$
\begin{aligned}
& \boldsymbol{E}\left[\left|X^{(i)}(s, t)\right|^{2} \leqq 5[3 \gamma(T ; Z)+1] \sum_{k=0}^{i}\left(5 B_{T} s t\right)^{k}(k !)^{-2}\right. \\
& \text { for } \quad(s, t) \prec(T, T)
\end{aligned}
$$

which holds not only for $i=1,2, \cdots$ but also for $i=0$ on account of (11). From (13) we have (4) by setting

$$
M_{T} \equiv 5[3 \gamma(T ; Z)+1] \sum_{k=0}^{\infty}\left(5 B_{T} T^{2}\right)^{k}(k !)^{-2}<\infty .
$$

This completes the proof of the lemma.

LEMMA 3.11. Under the same hypothesis as in Lemma 3.10, assume further that $\alpha$ and $\beta$ satisfy the Lipschitz condition (3.3) also. Then for every $T>0$ there exists $N_{T}>0$ such that

$$
\begin{aligned}
& \boldsymbol{E}\left[\sup _{(u, v)<(s t)}\left|(\tau X)(u, v)-\left(\tau X^{\prime}\right)(u, v)\right|^{2}\right] \\
& \leqq N_{T} \int_{[0, s] \times[0, t]} \sup _{\left(u^{\prime}, v^{\prime}\right)<(u, v)} \boldsymbol{E}\left[\left|X\left(u^{\prime}, v^{\prime}\right)-X^{\prime}\left(u^{\prime}, v^{\prime}\right)\right|^{2}\right] m_{L}(d(u, v)) \\
& \text { for }(s, t) \prec(T, T) \text { for any } X, X^{\prime} \in \boldsymbol{L}_{2}^{c}\left(\widetilde{\mho}_{s, t}\right) .
\end{aligned}
$$

Proof. Let $X, X^{\prime} \in \boldsymbol{L}_{2}^{c}\left(\mathfrak{F}_{s, t}\right)$. According to Lemma 3.10, $\tau X$ and $\tau X^{\prime}$ exist in $\boldsymbol{L}_{2}^{c}\left(\mathfrak{\mho}_{s, t}\right)$. Now from the defining equation (1) for $\tau$ in Lemma 3.10 we have

$$
\begin{aligned}
& \boldsymbol{E}\left[\sup _{(u, v)<(s, t)}\left|(\tau X)(u, v)-\left(\tau X^{\prime}\right)(u, v)\right|^{2}\right] \\
& \quad \leqq 2 \boldsymbol{E}\left[\sup _{(u, v)<(s, t)}|I(\Phi)(u, v)|^{2}\right]+2 \boldsymbol{E}\left[\sup _{(u, v)<(s, t)}|J(\Psi)(u, v)|^{2}\right]
\end{aligned}
$$

for $(s, t) \in D$ where

$$
\left\{\begin{array}{l}
\Phi(s, t, w)=\alpha(s, t, X(\cdot, \cdot, \omega))-\alpha\left(s, t, X^{\prime}(\cdot, \cdot, \omega)\right) \\
\Psi(s, t, w)=\beta(s, t, X(\cdot, \cdot, \omega))-\beta\left(s, t, X^{\prime}(\cdot, \cdot, \omega)\right)
\end{array}\right.
$$

and $I(\Phi)$ and $J(\Psi)$ are the stochastic integrals of $\Phi$ and $\Psi$ with respect to the $\widetilde{\mho}_{s, t}$ Brownian motion $B$ with $\partial B=0$ and the Lebesgue measure respectively. Note that by using (3.3) we have the same kind of estimate as (6) in the proof of Lemma 3.10 for our $\Phi$ and $\Psi$ to show that they are of the class $\mathfrak{R}_{2}\left(\mathfrak{F}_{s, t}\right)$ and this ensures the existence of the stochastic integrals $I(\Phi)$ and $J(\Psi)$.

Since $I(\Phi)$ is of the class $\mathfrak{M}_{2}^{c}\left(\mathfrak{\mho}_{s, t}\right)$, we have by the martingale inequality (b) in Theorem 1.2 of [3] with $p=2$ and then by (11) in the proof of Theorem 3.8 


$$
\begin{aligned}
E\left[\sup _{(u, v)<(s, t)}|I(\Phi)(u, v)|^{2}\right] & \leqq 16 \boldsymbol{E}\left[|I(\Phi)(s, t)|^{2}\right] \\
& =16 \boldsymbol{E}\left[\int_{[0, s] \times[0, t]}|\Phi(u, v)|^{2} m_{L}(d(u, v))\right] .
\end{aligned}
$$

For $J(\Psi)$ we have by the Schwarz Inequality

$$
\begin{aligned}
|J(\Psi)(u, v)|^{2} & =\left|\int_{[0, u] \times[0, v]} \Psi\left(u^{\prime}, v^{\prime}\right) m_{L}\left(d\left(u^{\prime}, v^{\prime}\right)\right)\right|^{2} \\
& \leqq u v \int_{[0, u] \times[0, v]}\left|\Psi\left(u^{\prime}, v^{\prime}\right)\right|^{2} m_{L}\left(d\left(u^{\prime}, v^{\prime}\right)\right) \\
& \leqq s t \int_{[0, s] \times[0, t]}\left|\Psi\left(u^{\prime}, v^{\prime}\right)\right|^{2} m_{L}\left(d\left(u^{\prime}, v^{\prime}\right)\right)
\end{aligned}
$$

for $(u, v) \prec(s, t)$ so that

$$
\boldsymbol{E}\left[\sup _{(u, v)<(s, t)}|J(\Psi)(u, v)|^{2}\right] \leqq s t \boldsymbol{E}\left[\int_{[0, s] \times[0, t]}|\Psi(u, v)|^{2} m_{L}(d(u, v))\right] .
$$

Using (3) and (4) in (1) and applying (3.3), we have

$$
\begin{aligned}
& \boldsymbol{E}\left[\sup _{(u, v)<(s, t)}\left|(\tau X)(u, v)-\left(\tau X^{\prime}\right)(u, v)\right|^{2}\right] \\
& \leqq\left(32+2 T^{2}\right) \boldsymbol{E}\left[\int_{[0, s] \times[0, t]}\left\{|\Phi(u, v)|^{2}+|\Psi(u, v)|^{2}\right\} m_{L}(d(u, v))\right] \\
& \leqq\left(32+2 T^{2}\right) L_{T} \boldsymbol{E}\left[\int _ { [ 0 , s ] \times [ 0 , t ] } \left\{\int_{[0, u] \times[0, v]}\left|X\left(u^{\prime}, v^{\prime}\right)-X^{\prime}\left(u^{\prime}, v^{\prime}\right)\right|^{2}\right.\right. \\
&\left.\left.\times \lambda\left(d\left(u^{\prime}, v^{\prime}\right)\right)+\left|X(u, v)-X^{\prime}(u, v)\right|^{2}\right\} m_{L}(d(u, v))\right] \\
& \leqq\left(32+2 T^{2}\right) L_{T}\{\lambda([0, T] \times[0, T])+1\} \\
& \times \int_{[0, s] \times[0, t]} \sup _{\left\{u^{\prime}, v^{\prime}\right)<\langle u, v)} \boldsymbol{E}\left[\left|X\left(u^{\prime}, v^{\prime}\right)-X^{\prime}\left(u^{\prime}, v^{\prime}\right)\right|^{2}\right] m_{L}(d(u, v)) .
\end{aligned}
$$

If we let

$$
N_{T}=\left(32+T^{2}\right) L_{T}\{\lambda([0, T] \times[0, T])+1\},
$$

then the lemma is proved.

THEOREM 3.12. Suppose the coefficients $\alpha$ and $\beta$ in the stochastic differential equation (3.1) in Definition 3.3 satisfy the conditions (3.3) and (3.4). Let $\left(\Omega, \mathfrak{\mho}, P\right.$; $\left.\mathfrak{\mho}_{s, t}\right)$ be an equipped probability space on which an $\mathfrak{\mho}_{s, t}$ Brownian motion $B$ with $\partial B=0$ exists. Then for every $Z \in \boldsymbol{L}_{2}^{c}\left(\mathfrak{\mho}_{s, t} \mid \partial D\right)$ there exists a strong solution $(X, B)$ of $(3.1)$ in which $X \in \boldsymbol{L}_{2}^{c}\left(\mathfrak{\mho}_{s, t}\right)$ and $\partial X=Z$. In fact there exists a transformation $F$ of $\partial W \times W$ into $W$ satisfying $1^{\circ}$ and $2^{\circ}$ of Definition 3.6 such that

$$
X(\cdot, \cdot, \omega)=F[Z(\cdot, \cdot, \omega), B(\cdot, \cdot, \omega)] \text { for a.e. } \omega \in \Omega
$$


for all $Z \in \boldsymbol{L}_{2}^{c}\left(\mathfrak{\mho}_{s, t} \mid \partial D\right)$ and $X \in \boldsymbol{L}_{2}^{c}\left(\mathfrak{\mho}_{s, t}\right)$ in the corresponding solutions $(X, B)$ of (3.1) with $\partial X=Z$.

(Recall that according to Theorem 3.8 the condition $\partial X=Z$ determines $X$ in the solution $(X, B)$ uniquely up to a null set in $(\Omega, \Im, P)$.)

Proof (1). Let $Z \in \boldsymbol{L}_{2}^{c}\left(\widetilde{\mho}_{s, t} \mid \partial D\right)$. Define a sequence $\left\{X^{(i)}, i=\right.$ $0,1,2, \cdots\}$ in $\boldsymbol{L}_{2}^{c}\left(\mathfrak{\mho}_{s, t}\right)$ as in Lemma 3.10 by

$$
\left\{\begin{array}{l}
X^{(0)}(s, t)=-Z(0,0)+Z(s, 0)+Z(0, t) \text { for }(s, t) \in D, \\
X^{(i)}(s, t)=\left(\tau X^{(i-1)}\right)(s, t) \text { for }(s, t) \in D \text { and } i=1,2, \cdots .
\end{array}\right.
$$

By an iterated application of Lemma 3.11 and by the fact that for any $\mathfrak{F}_{s, t}$ adapted process $Y$ on $\left(\Omega, \mathfrak{F}, P ; \mathfrak{F}_{s, t}\right)$ the inequality

$$
\sup _{(u, v)<(s, t)} E[|Y(u, v)|] \leqq E\left[\sup _{(u, v)<(s, t)}|Y(u, v)|\right] \quad \text { for } \quad(s, t) \in D
$$

holds, we have for every $T>0$ some $N_{T}>0$ such that

$$
\begin{aligned}
& E\left[\sup _{(s, t)<(T, T)}\left|X^{(i+1)}(s, t)-X^{(i)}(s, t)\right|^{2}\right] \\
& \leqq N_{T}^{i} \int_{[0, T] \times[0, T]} \int_{\left[0, s_{1}\right] \times\left[0, t_{1}\right]} \cdots \int_{\left[0, s_{i-1}\right] \times\left[0, t_{i-1}\right]} \\
& \times \sup _{(u, v)<\left(s_{i}, t_{i}\right)} E\left[\left|X^{(1)}(u, v)-X^{(0)}(u, v)\right|^{2}\right] m_{L}\left(d\left(s_{i}, t_{i}\right)\right) \cdots \\
& \quad m_{L}\left(d\left(s_{2}, t_{2}\right)\right) m_{L}\left(d\left(s_{1}, t_{1}\right)\right) .
\end{aligned}
$$

Since $\left(s_{i}, t_{i}\right) \prec\left(s_{i-1}, t_{i-1}\right) \prec \cdots \prec\left(s_{1}, t_{1}\right) \prec(T, T)$ in the integral above, we have by (4) of Lemma 3.10

$$
\sup _{\langle u, v)<\left(s_{i}, t_{i}\right)} \boldsymbol{E}\left[\left|X^{(1)}(u, v)-X^{(0)}(u, v)\right|^{2}\right] \leqq 4 M_{T} .
$$

Using (4) and (3) and integrating we have

$$
\boldsymbol{E}\left[\sup _{(s, t)<(T, T)}\left|X^{(i+1)}(s, t)-X^{(i)}(s, t)\right|^{2}\right] \leqq 4 M_{T} N_{T}^{i} T^{2 i}(i !)^{-2} .
$$

Let

$$
\begin{array}{r}
A_{i}=\left\{\omega \in \Omega ; \sup _{(s, t)<(T, T)}\left|X^{(i+1)}(s, t)-X^{(i)}(s, t)\right|>2^{-i}\right\} \\
\text { for } i=0,1,2, \cdots .
\end{array}
$$

Then by the Chebyshev Inequality and (5)

$$
P\left(A_{i}\right) \leqq 16 M_{T} N_{T}^{2} T^{2 i}(i !)^{-2} \text { for } i=0,1,2, \cdots .
$$

Since $\sum_{i=0}^{\infty} N_{T}^{i} T^{2 i}(i !)^{-2}<\infty$, the Borel-Cantelli lemma applies and consequently $\left\{X^{(i)}, i=0,1,2, \cdots\right\}$ converges uniformly for $(s, t) \prec$ $(T, T)$ for a.e. $\omega \in \Omega$. By letting $T=1,2, \cdots$, we conclude that 
there exists a null set $N$ in $(\Omega, \Im, P)$ such that $\left\{X^{(i)}, i=0,1,2, \cdots\right\}$ converges on $D$ for each $\omega \in N^{c}$ where the convergence is uniform on every bounded subset of $D$ for each $\omega \in N^{c}$.

Let us define a stochastic process $X$ on $\left(\Omega, \mathfrak{\mho}, P ; \mathfrak{\mho}_{s, t}\right)$ by

$$
X(s, t, \omega)=\left\{\begin{array}{lll}
\lim _{i \rightarrow \infty} X^{(i)}(s, t, \omega) & \text { for }(s, t) \in D \text { and } \omega \in N^{c}, \\
0 & \text { for }(s, t) \in D \text { and } \omega \in N .
\end{array}\right.
$$

Then every sample function of $X$ is continuous on $D$. Also, since $X^{(i)}$ is $\mathfrak{\mho}_{s, t}$ adapted and $N \in \mathfrak{\mho}_{s, t}$ for every $(s, t) \in D, X$ is $\mathfrak{\mho}_{s, t}$ adapted. To show that $X \in \boldsymbol{L}_{2}^{c}\left(\mathfrak{\mho}_{s, t}\right)$ it remains to show that for every $T>0$

$$
\sup _{(s, t)<(T, T)} \boldsymbol{E}\left[|X(s, t)|^{2}\right]<\infty .
$$

Now for the $L_{2}$ norm $\boldsymbol{E}\left[|\cdot|^{2}\right]^{1 / 2}$ on the Hilbert space $L_{2}(\Omega, \mathfrak{F}, P)$ we have by the triangle inequality and by (2) and (5)

$$
\begin{aligned}
& \sup _{(s, t)<(T, T)} E\left[\left|X^{(m)}(s, t)-X^{(n)}(s, t)\right|^{2}\right]^{1 / 2} \\
& \leqq \sum_{i=n}^{m-1} \sup _{(s, t)<(T, T)} E\left[\left|X^{(i+1)}(s, t)-X^{(i)}(s, t)\right|^{2}\right]^{1 / 2} \\
& \leqq 2 \sqrt{M_{T}} \sum_{i=n}^{m-1} \sqrt{N_{T}^{i}} T^{i}(i !)^{-1} \quad \text { for } \quad(s, t) \in D .
\end{aligned}
$$

Since $\sum_{i=0}^{\infty} \sqrt{N_{T}^{i}} T^{i}(i !)^{-1}<\infty$, the Cauchy Criterion for Uniform Convergence implies the existence of an $\mathfrak{\mho}_{s, t}$ adapted process $X^{*}$ on $\left(\Omega, \mathfrak{F}, P ; \mathfrak{\mho}_{s, t}\right)$ such that

$$
\left.\boldsymbol{E}\left[\mid X^{*}(s, t)\right]\right|^{2}<\infty \quad \text { for } \quad(s, t) \in D
$$

and

$$
\lim _{i \rightarrow \infty}\left\{\sup _{(s, t)<(T, T)} E\left[\left|X^{(i)}(s, t)-X^{*}(s, t)\right|^{2}\right]\right\}=0 .
$$

From (10) and the fact that $X^{(i)} \in \boldsymbol{L}_{2}^{c}\left(\widetilde{\mho}_{s, t}\right)$ we have

$$
\begin{aligned}
\sup _{(s, t)<(T, T)} \boldsymbol{E}\left[\left|X^{*}(s, t)\right|^{2}\right] \leqq & 2 \sup _{(s, t)<\left(T^{T}, T\right)} \boldsymbol{E}\left[\left|X^{*}(s, t)-X^{(i)}(s, t)\right|^{2}\right. \\
& +2 \sup _{(s, t)<(T, T)} \boldsymbol{E}\left[\left|X^{(i)}(s, t)\right|^{2}\right]<\infty .
\end{aligned}
$$

Since convergence in $L_{2}$ norm implies the existence of an a.e. convergent subsequence, (6) and (10) imply

$$
X(s, t, \omega)=X^{*}(s, t, \omega) \text { for a.e. } \omega \in \Omega \text { for each }(s, t) \in D .
$$

By (11) and (12) we have (7). This shows that $X \in \boldsymbol{L}_{2}^{c}\left(\widetilde{\mho}_{s, t}\right)$.

Now that $X \in \boldsymbol{L}_{2}^{c}\left(\mathfrak{\mho}_{s, t}\right), \tau X$ exists in $\boldsymbol{L}_{2}^{c}\left(\mathfrak{\mho}_{s, t}\right)$ by Lemma 3.10. We proceed to show that in fact $\tau X=X$. Now for every $T>0$ 


$$
\begin{aligned}
\sup _{(s, t)<(T, T)}|(\tau X)(s, t)-X(s, t)|^{2} \leqq & 2 \sup _{(s, t)<(T, T)}\left|(\tau X)(s, t)-X^{(i+1)}(s, t)\right|^{2} \\
& +2 \sup _{(s, t)<(T, T)}\left|X^{(i+1)}(s, t)-X(s, t)\right|^{2}
\end{aligned}
$$

for each $i$. Since $\left\{X^{(i)}, i=0,1,2, \cdots\right\}$ converges uniformly on every bounded subset of $D$ for each $\omega \in N^{c}$ to $X$, we have

$$
\lim _{i \rightarrow \infty}\left\{\sup _{(s, t)<(T, T)}\left|X^{(i+1)}(s, t, \omega)-X(s, t, \omega)\right|^{2}\right\}=0 \quad \text { for } \omega \in N^{c} .
$$

This implies

$$
\begin{aligned}
\sup _{(s, t)<(T, T)} \mid & (\tau X)(s, t, \omega)-\left.X(s, t, \omega)\right|^{2} \\
& \leqq 2 \liminf _{i \rightarrow \infty}\left\{\sup _{(s, t)<(T, T)}\left|(\tau X)(s, t, \omega)-X^{(i+1)}(s, t, \omega)\right|^{2}\right\}
\end{aligned}
$$

for $\omega \in N^{c}$.

Taking the expectation on both sides of inequality above, then applying Fatou's Lemma and Lemma 3.11 to the right side we have

$$
\begin{aligned}
E\left[\sup _{(s, t)<(T, T)}\right. & \left.|(\tau X)(s, t)-X(s, t)|^{2}\right] \\
\leqq & 2 N_{T} \liminf _{i \rightarrow \infty} \int_{[0, T] \times[0, T]} \sup _{(u, v)<(s, t)} E\left[\left|X(u, v)-X^{(i)}(u, v)\right|^{2}\right] \\
& \times m_{L}(d(s, t)) .
\end{aligned}
$$

Now by Definition 3.9 and (4) of Lemma 3.10

$$
\sup _{(u, v)<(s, t)} E\left[\left|X(u, v)-X^{(i)}(u, v)\right|^{2}\right] \leqq 2 \gamma(T ; X)+2 M_{T} .
$$

Let us replace lim inf $f_{i \rightarrow \infty}$ on the right side of (13) by $\lim \sup _{i \rightarrow \infty}$. The inequality remains valid. Since the integrand on the right side of (13) is bounded by a constant as we have just shown, Fatou's lemma for lim sup applies and we have

$$
\begin{aligned}
& \boldsymbol{E}\left[\sup _{(s, t)<(T, T)}|(\tau X)(s, t)-X(s, t)|^{2}\right] \\
& \quad \leqq 2 N_{T} \int_{[0, T] \times[0, T]} \limsup _{i \rightarrow \infty}\left\{\sup _{(u, v)<(s, t)} \boldsymbol{E}\left[\left|X(u, v)-X^{(i)}(u, v)\right|^{2}\right]\right\} m_{L}(d(s, t))
\end{aligned}
$$

which is equal to 0 by (12) and (10). Therefore

$$
\sup _{(s, t)<(T, T)}|(\tau X)(s, t, \omega)-X(s, t, \omega)|=0 \quad \text { for a.e. } \omega \in \Omega .
$$

By considering $T=1,2, \cdots$, we conclude that

$$
(\tau X)(s, t, \omega)=X(s, t, \omega) \text { for all }(s, t) \in D \text { for a.e. } \omega \in \Omega \text {. }
$$

Thus we have shown that $\tau X=X$.

Recalling the definition of $\tau$ by (1) of Lemma 3.10, we have 


$$
\begin{aligned}
X(s, t)= & -Z(0,0)+Z(s, 0)+Z(0, t) \\
& +\int_{[0, s] \times[0, t]} \alpha(u, v, X) d B(u, v) \\
& +\int_{[0, s] \times[0, t]} \beta(u, v, X) d(u, v),
\end{aligned}
$$

i.e., $(X, B)$ is a solution of the stochastic differential equation (3.1) on $\left(\Omega, \mathfrak{F}, P ; \mathfrak{F}_{s, t}\right)$ with $\partial X=Z$. Bh Theorem $3.8,(X, B)$ is the unique solution on $\left(\Omega, \mathfrak{\mho}, P ; \mathfrak{\mho}_{s, t}\right)$ with $\partial X=Z$.

(2) We proceed to show that there exists a transformation $F$ of $\partial W \times W$ into $W$ satisfying $1^{\circ}$ and $2^{\circ}$ of Definition 3.6 such that for every $Z \in \boldsymbol{L}_{2}^{c}\left(\mathfrak{F}_{s, t} \mid \partial D\right)$ the corresponding stochastic process $X \in$ $\boldsymbol{L}_{2}^{(}\left(\widetilde{\mho}_{s, t}\right)$ defined by (1) and (6) satisfies

$$
X(\cdot, \cdot, \omega)=F[Z(\cdot, \cdot, \omega), B(\cdot, \cdot, \omega)] \text { for a.e. } \omega \in \Omega
$$

where the exceptional null set in $(\Omega, \Im, P)$ may depend on $Z$.

We shall show first that for every $i=0,1,2, \cdots$, there exists a transformation $F^{(i)}$ of $\partial W \times W$ into $W$ satisfying $1^{\circ}$ and $2^{\circ}$ of Definition 3.6 such that for every $Z \in \boldsymbol{L}_{2}^{c}\left(\widetilde{\mho}_{s, t} \mid \partial D\right)$ the corresponding stochastic process $X^{(i)} \in \boldsymbol{L}_{2}\left(\widetilde{\mho}_{s, t}\right)$ defined by (1) satisfies

$$
X^{(i)}(\cdot, \cdot, \omega)=F^{(i)}[Z(\cdot, \cdot, \omega), B(\cdot, \cdot, \omega)] \text { for a.e. } \omega \in \Omega
$$

where the exceptional null set in $(\Omega, \mathfrak{F}, P)$ may depend on $Z$. We shall show this below by induction on $i$.

(2.1) Let us show the existence of $F^{(0)}$. Recall that by (1),

$$
\begin{array}{r}
X^{(0)}(s, t, \omega)=-Z(0,0, \omega)+Z(s, 0, \omega)+Z(0, t, \omega) \\
\text { for }(s, t, \omega) \in D \times \Omega .
\end{array}
$$

Consider the following transformations:

$$
\begin{aligned}
& \pi_{0} ; \quad \partial W \rightarrow \boldsymbol{R} \quad \text { defined by } \pi_{0} z=z(0,0) \in \boldsymbol{R} \text { for } z \in \partial W, \\
& \pi_{1} ; \quad \partial W \rightarrow W^{(1)} \quad \text { defined by } \pi_{1} z=z(\cdot, 0) \in W^{(1)} \text { for } z \in \partial W \text {, } \\
& \pi_{2} ; \quad \partial W \rightarrow W^{(1)} \quad \text { defined by } \pi_{2} z=z(0, \cdot) \in W^{(1)} \text { for } z \in W .
\end{aligned}
$$

Thus defined, $\pi_{0}$ is $\mathfrak{B}(\partial W) / \mathfrak{B}(\boldsymbol{R})$ measurable and $\pi_{1}$ and $\pi_{2}$ are both $\mathfrak{B}(\partial W) / \mathfrak{B}\left(W^{(1)}\right)$ measurable. Consider the following transformations:

$$
\begin{aligned}
& e_{0} ; \quad \boldsymbol{R} \rightarrow W \text { defined by } e_{0} \zeta=w_{\zeta} \\
& \text { where } w_{\zeta}(s, t)=\zeta \text { for }(s, t) \in D \text {, } \\
& e_{1} ; \quad W^{(1)} \rightarrow W \quad \text { defined by } e_{1} x=w_{x} \\
& \text { where } w_{x}(s, t)=x(s) \text { for }(s, t) \in D \text {, } \\
& e_{2} ; \quad W^{(1)} \rightarrow W \quad \text { defined by } e_{1} y=w_{y} \\
& \text { where } w_{y}(s, t)=y(t) \text { for }(s, t) \in D \text {. }
\end{aligned}
$$


Clearly $e_{0}$ is $\mathfrak{B}(\boldsymbol{R}) / \mathfrak{B}(W)$ measurable and $e_{1}$ and $e_{2}$ are both $\mathfrak{B}\left(W^{(1)}\right) / \mathfrak{B}(W)$ measurable. Thus $e_{0} \circ \pi_{0}, e_{1} \circ \pi_{1}$ and $e_{2} \circ \pi_{2}$ are all $\mathfrak{B}(\partial W) / \mathfrak{B}(W)$ measurable transformations of $\partial W$ into $W$. Define a transformation $F^{(0)}$ of $\partial W \times W$ into $W$ by

$$
F^{(0)}(z, w)=-\left(e_{0} \circ \pi_{0}\right) z+\left(e_{1} \circ \pi_{1}\right) z+\left(e_{2} \circ \pi_{2}\right) z \text { for }(z, w) \in \partial W \times W .
$$

Then $F^{(0)}$ is $\sigma(\mathfrak{B}(\partial W) \times \mathfrak{B}(W)) / \mathfrak{B}(W)$ measurable and $1^{\circ}$ and $2^{\circ}$ of Definition 3.6 are satisfied trivially. Also

$$
\begin{aligned}
& F^{(0)}[Z(\cdot, \cdot, w), B(\cdot, \cdot, w)] \\
& \quad=-\left(e_{0} \circ \pi_{0}\right) Z(\cdot, \cdot, \omega)+\left(e_{1} \circ \pi_{1}\right) Z(\cdot, \cdot, \omega)+\left(e_{2} \circ \pi_{2}\right) Z(\cdot, \cdot, \omega) \\
& \quad=X^{(0)}(\cdot, \cdot, \omega) \text { for } \omega \in \Omega .
\end{aligned}
$$

This proves the existence of $F^{(0)}$.

(2.2) Next suppose that for some $i$ there exists a transformation $F^{(i)}$ of $\partial W \times W$ into $W$ satisfying $1^{\circ}$ and $2^{\circ}$ of Definition 3.6 and (16). Now by (1) and the definition of $\tau$ in Lemma 3.10,

$$
X^{(i+1)}(s, t)=X^{(0)}(s, t)+I\left(\Phi^{(i)}\right)(s, t)+J\left(\Psi^{(i)}\right)(s, t)
$$

where

$$
\left\{\begin{array}{l}
\Phi^{(i)}(s, t, \omega)=\alpha\left[s, t, X^{(i)}(\cdot, \cdot, \omega)\right] \text { for } \quad(s, t, \omega) \in D \times \Omega, \\
\Psi^{(i)}(s, t, \omega)=\beta\left[s, t, X^{(i)}(\cdot, \cdot, \omega)\right] \text { for } \quad(s, t, \omega) \in D \times \Omega,
\end{array}\right.
$$

and $I\left(\Phi^{(i)}\right)$ and $J\left(\Psi^{(i)}\right)$ are the stochastic integrals of $\Phi^{(i)}$ and $\Psi^{(i)}$ with respect to the $\mathfrak{F}_{s, t}$ Brownian motion $B$ with $\partial B=0$ and the Lebesgue measure respectively. Since we have (17) already, if we show that there exist transformations $G^{(i)}$ and $H^{(i)}$ of $\partial W \times W$ into $W$ satisfying $1^{\circ}$ and $2^{\circ}$ of Definition 3.6 such that

$$
\left\{\begin{array}{l}
I\left(\Phi^{(i)}\right)(\cdot, \cdot)=G^{(i)}[Z(\cdot, \cdot, \omega), B(\cdot, \cdot, \omega)] \text { for a.e. } \omega \in \Omega, \\
J\left(\Psi^{(i)}\right)(\cdot, \cdot)=H^{(i)}[Z(\cdot, \cdot, \omega), B(\cdot, \cdot, \omega)] \text { for a.e. } \omega \in \Omega,
\end{array}\right.
$$

for $X^{(i)}$ defined by (1) with an arbitrary $Z \in \boldsymbol{L}_{2}^{c}\left(\Im_{s, t} \mid \partial D\right)$, then the transformation $F^{(i+1)}$ of $\partial W \times W$ into $W$ defined by

$$
F^{(i+1)}=F^{(0)}+G^{(i)}+H^{(i)}
$$

satisfies $1^{\circ}$ and $2^{\circ}$ of Definition 3.6 and also

$$
X^{(i+1)}(\cdot, \cdot, \omega)=F^{(i+1)}[Z(\cdot, \cdot, \omega), B(\cdot, \cdot, \omega)] \text { for a.e. } \omega \in \Omega .
$$

It remains to prove the existence of $G^{(i)}$ and $H^{(i)}$. We do this for $G^{(2)}$ below.

Now according to our induction hypothesis, (16) holds for our $i$. Then by (19)

$$
\begin{aligned}
& \Phi^{(i)}(s, t, \omega)=\alpha\left(s, t, F^{(i)}[Z(\cdot, \cdot \omega), B(\cdot, \cdot, \omega)]\right) \\
& \quad \text { for }(s, t) \in D \text { and a.e. } \omega \in \Omega .
\end{aligned}
$$


Since $X^{(i)} \in \boldsymbol{L}_{2}^{c}\left(\widetilde{\mho}_{s, t}\right)$, our $\Phi^{(i)}$ defined by (19) is of the class $\mathfrak{\Omega}_{2}\left(\mathfrak{\mho}_{s, t}\right)$ as we saw in the proof of Lemma 3.10. Let us consider first the particular case where $\alpha$ is such that $\Phi^{(i)}$ is of the class $\mathfrak{L}_{0}\left(\mathfrak{\mho}_{s, t}\right)$. Then by Definition 2.8 for some null set $N$ in $(\Omega, \mathfrak{F}, P)$

$$
I\left(\Phi^{(i)}\right)(s, t, \omega)=\left\{\begin{aligned}
\sum_{j=1}^{m} \sum_{k=1}^{n} \alpha\left(s_{j-1}, t_{k-1}, F^{(i)}[Z(\cdot, \cdot \omega), B(\cdot, \cdot \omega)]\right) \\
\quad \times\left\{B\left(s_{j}, t_{k}, \omega\right)-B\left(s_{j-1}, t_{k}, \omega\right)-B\left(s_{j}, t_{k-1} \omega\right)\right. \\
\left.+B\left(s_{j-1}, t_{k-1}, \omega\right)\right\} \text { for }(s, t, \omega) \in D \times N^{c}, \\
0 \quad \text { for }(s, t, \omega) \in D \times N,
\end{aligned}\right.
$$

with the understanding that $s_{m}=s$ and $t_{n}=t$ as in Definition 2.8. Define transformations $\varphi$ and $\iota(\varphi)$ of $D \times \partial W \times W$ into $\boldsymbol{R}$ by

$$
\begin{aligned}
& \varphi(s, t, z, w)=\alpha\left(s, t, F^{(i)}[z, w]\right) \text { for }(s, t, z, w) \in D \times \partial W \times W \\
& \iota(\varphi)(s, t, z, w) \\
& =\sum_{j=1}^{m} \sum_{k=1}^{n} \alpha\left(s_{j-1}, t_{k-1}, F^{(i)}[z, w]\right)\left\{W\left(s_{j}, t_{k}\right)-w\left(s_{j-1}, t_{k}\right)\right. \\
& \left.\quad-W\left(s_{j}, t_{k-1}\right)+w\left(s_{j-1}, t_{k-1}\right)\right\} \text { for }(s, t, z, w) \in D \times \partial W \times W,
\end{aligned}
$$

again with the understanding that $s_{m}=s$ and $t_{n}=t$. Let us show that the transformation $c(\varphi)(\cdot, \cdot, z, w)$ of $(z, w) \in \partial W \times W$ into $W$ is $\sigma(\mathfrak{B}(\partial W) \times \mathfrak{B}(W)) / \mathfrak{B}(W)$ measurable. According to part (1) of Proposition 3.1 it suffices to show that for every $(s, t) \in D$ the transformation $\iota(\varphi)(s, t, z, w)$ of $(z, w) \in \partial W \times W$ into $\boldsymbol{R}$ is $\sigma(\mathfrak{B}(\partial W) \times \mathfrak{B}(W)) / \mathfrak{B}(\boldsymbol{R})$ measurable. Now $1^{\circ}$ of Definition 3.6 and $2^{\circ}$ of Definition 3.2 imply that the transformation $\alpha\left(s_{j-1}, t_{k-1}, F^{(i)}[z, w]\right)$ of $(z, w) \in \partial W \times W$ into $\boldsymbol{R}$ is $\sigma(\mathfrak{B}(\partial W) \times \mathfrak{B}(W)) / \mathfrak{B}(\boldsymbol{R})$ measurable. Also the second factor in each summand on the right side of $(26)$ is a $\mathfrak{B}(W) / \mathfrak{B}(\boldsymbol{R})$ measurable transformation of $W$ into $\boldsymbol{R}$. Therefore the transformation $\iota(\varphi)(s, t, z, w)$ of $(\boldsymbol{z}, w) \in \partial W \times W$ into $\boldsymbol{R}$ is $\sigma(\mathfrak{B}(\partial W) \times \mathfrak{B}(W)) / \mathfrak{B}(\boldsymbol{R})$ measurable. Similarly the fact that for each $z \in \partial W$ the transformation $\iota(\varphi)(\cdot, \cdot, z, w)$ of $w \in W$ into $W$ is $\mathfrak{B}_{s, t}(W) / \mathfrak{B}_{s, t}(W)$ measurable for every $(s, t) \in D$ can be shown by part (2) of Proposition $3.1,2^{\circ}$ of Definition 3.6 and $2^{\circ}$ of Definition 3.2. With these two measurability conditions satisfied by $c(\varphi)$ we conclude that there exists a transformation $G^{(i)}$ of $\partial W \times W$ into $W$ satisfying $1^{\circ}$ and $2^{\circ}$ of Definition 3.6 such that

$$
\iota(\varphi)(\cdot, \cdot, z, w)=G^{(i)}(z, w) \text { for }(z, w) \in \partial W \times W .
$$

From (24), (26) and (27) we have (20) for $\Phi^{(i)}$ for the particular case where $\alpha$ is such that $\Phi^{(i)}$ is of the class $\mathfrak{Z}_{0}\left(\widetilde{\jmath}_{s, t}\right)$.

Consider now the general case where $\Phi^{(i)}$ is of the class $\Omega_{2}\left(\widetilde{\mho}_{s, t}\right)$. According to (2.1) in $\S 2$ and Proposition 1.6 there exist a sequence 
$\left\{\Phi_{l}, l=1,2, \cdots\right\}$ in the class $\mathfrak{Z}_{0}\left(\widetilde{\mho}_{s, t}\right)$ and a null set $N_{0}$ in $(\Omega, \mathfrak{F}, P)$ such that

$$
\begin{array}{ll}
\lim _{l \rightarrow \infty} I\left(\Phi_{l}\right)(s, t, \omega)=I\left(\Phi^{(i)}\right)(s, t, \omega) & \text { uniformly on every bounded } \\
& \text { subset of } D \text { for } \omega \in N_{0}^{c},
\end{array}
$$

i.e.,

(28) $\lim _{l \rightarrow \infty} I\left(\Phi_{l}\right)(\cdot, \cdot, \omega)=I\left(\Phi^{(i)}\right)(\cdot, \cdot, \omega)$ in the metric topology of $W$ for $\omega \in N_{0}^{c}$.

Since $\Phi_{l}$ is of the class $\mathfrak{R}_{0}\left(\widetilde{\mho}_{s, t}\right)$, there exists a transformation $G_{l}^{(i)}$ of $\partial W \times W$ into $W$ satisfying $1^{\circ}$ and $2^{\circ}$ of Definition 3.6 and a null set $N_{l}$ in $(\Omega, \widetilde{F}, P)$ such that

$$
I\left(\Phi_{l}\right)(\cdot, \cdot \omega)=G_{l}^{(i)}[Z(\cdot, \cdot, \omega), B(\cdot, \cdot, \omega)] \text { for } \omega \in N_{l}^{c} .
$$

Let

$$
N=\bigcup_{l=0}^{\infty} N_{l}
$$

and

$$
\Lambda=\left\{(z, w) \in \partial W \times W ; \lim _{l \rightarrow \infty} G_{l}^{(i)}[z, w] \text { exists in } W\right\} .
$$

From the $\sigma(\mathfrak{B}(\partial W) \times \mathfrak{B}(W)) / \mathfrak{B}(W)$ measurability of $G_{l}^{(i)}$ for $l=1,2, \cdots$, $\Lambda \in \sigma(\mathfrak{B}(\partial W) \times \mathfrak{B}(W))$. Define a transformation $G^{(i)}$ of $\partial W \times W$ into $W$ by

$$
G^{(i)}(z, w)=\left\{\begin{array}{l}
\lim _{l \rightarrow \infty} G_{l}^{(i)}(z, w) \text { for }(z, w) \in \Lambda, \\
\text { a fixed element in } W \text { for }(z, w) \in \Lambda^{c} .
\end{array}\right.
$$

Then $G^{(i)}$ satisfies $1^{\circ}$ and $2^{\circ}$ of Definition 3.6 since $G_{l}^{(i)}$ does for $l=$ $1,2, \cdots$. Let $K$ be the subset of $\partial W \times W$ covered by the transformation of $N^{c}$ defined by $[Z(\cdot, \cdot, \omega), B(\cdot, \cdot, \omega)]$ for $\omega \in N^{c}$. Then for every $(z, w) \in K$ there exists $\omega \in N^{c}$ such that

$$
(z, w)=[Z(\cdot, \cdot, \omega), B(\cdot, \cdot, \omega)]
$$

so that by (29), (28) and (30)

$$
\begin{aligned}
\lim _{l \rightarrow \infty} G_{l}^{(i)}(z, w) & =\lim _{l \rightarrow \infty} G_{l}^{(i)}[Z(\cdot, \cdot, \omega), B(\cdot, \cdot, \omega)] \\
& =\lim _{l \rightarrow \infty} I\left(\Phi_{l}\right)(\cdot, \cdot, \omega)=I\left(\Phi^{(i)}\right)(\cdot, \cdot \omega) \quad \text { for } \quad(z, w) \in K .
\end{aligned}
$$

Then by (31) $K \subset \Lambda$ so that by (32)

$$
\begin{aligned}
& \lim _{l \rightarrow \infty} G_{l}^{(i)}[Z(\cdot, \cdot, \omega), B(\cdot, \cdot \omega)] \\
& =G^{(i)}[Z(\cdot, \cdot \omega), B(\cdot, \cdot, \omega)] \text { for } \omega \in N^{c} .
\end{aligned}
$$


Combining (28), (29) and (33) we have

$$
I\left(\Phi^{(i)}\right)(\cdot, \cdot, \omega)=G^{(2)}[Z(\cdot, \cdot \omega), B(\cdot, \cdot, \omega)] \text { for } \omega \in N^{c},
$$

proving (20) for $I\left(\Phi^{(2)}\right)$. We can prove (20) for $J\left(\Psi^{(i)}\right)$ in the same way. This completes the proof that if $F^{(2)}$ exists so does $F^{(2+1)}$.

(2.3) By induction on $i$ we conclude that for each $i=0,1,2, \cdots$, there exists a transformation $F^{(2)}$ of $\partial W \times W$ into $W$ satisfying $1^{\circ}$ and $2^{\circ}$ of Definition 3.6 such that for any $Z \in \boldsymbol{L}_{2}^{c}\left(\widetilde{\mho}_{s, t} \mid \partial D\right)$ and the corresponding $X^{(\imath)} \in \boldsymbol{L}_{2}^{c}\left(\mathfrak{\mho}_{s, t}\right)$ defined by (1) we have

$$
X^{(i)}(\cdot, \cdot, \omega)=F^{(\imath)}[Z(\cdot, \cdot, \omega), B(\cdot, \cdot, \omega)] \text { for } \omega \in \Omega-N_{i}(Z)
$$

where $N_{i}(Z)$ is a null set in $(\Omega, \mathfrak{F}, P)$. Let

$$
N(Z)=\bigcup_{\imath=0}^{\infty} N_{\imath}(Z)
$$

and

$$
A=\left\{(z, w) \in \hat{\partial} W \times W ; \lim _{\imath \rightarrow \infty} F^{(i)}(z, w) \text { exists in } W\right\} .
$$

From the $\sigma(\mathfrak{B}(\partial W) \times \mathfrak{B}(W)) / \mathfrak{B}(W)$ measurability of $F^{(i)}, i=0,1,2, \cdots$, $\Lambda \in \sigma(\mathfrak{B}(\partial W) \times \mathfrak{B}(W))$. Define a transformation $F$ of $\partial W \times W$ into $W$ by

$$
F(z, w)=\left\{\begin{array}{l}
\lim _{i \rightarrow \infty} F^{(i)}(z, w) \text { for }(z, w) \in \Lambda, \\
\text { a fixed element in } W \text { for }(z, w) \in \Lambda^{c}
\end{array}\right.
$$

Then $F$ satisfies $1^{\circ}$ and $2^{\circ}$ of Definition 3.6 since $F^{(i)}$ does for $i=$ $0,1,2, \cdots$. We saw in (1) that

$$
\begin{gathered}
\lim _{\imath \rightarrow \infty} X^{(i)}(\cdot, \cdot, \omega)=X(\cdot, \cdot, \omega) \quad \text { in the metric topology of } W \\
\text { for } w \in N^{c}
\end{gathered}
$$

where $N$ is a null set in $(\Omega, \widetilde{F}, P)$. By the same argument as in proving (34) by means of (28), (29), (31) and (32) we conclude from (39), (35), (36), (37), and (38)

$$
X(\cdot, \cdot \omega)=F[Z(\cdot, \cdot, \omega), B(\cdot, \cdot, \omega)] \text { for } \omega \in(N(Z) \cup N)^{c} .
$$

This completes the proof of the theorem.

\section{REFERENCES}

1. R. Cairoli, Une inégalité pour martingales à indices multiples et ses applications, Lecture Notes in Mathematics, 124 (1970), 1-27, Springer-Verlag, Berlin.

2. - Sur une équation différentielle stochastique, C.R. Acad. Sc. Paris Ser. A, 274 (1972), 1739-1742.

3. R. Cairoli and J. B. Walsh, Stochastic integrals in the plane, Acta Math., 134 (1975), 111-183. 
4. J. L. Doob, Stochastic Processes, John Wiley, New York, 1953.

5. K. Ito, Lectures on Stochastic Processes, Tata Institute, Bombay, 1961.

6. H. Kunita and S. Watanabe, On square integrable martingales, Nagoya Math. J., 30 (1967), 209-245.

7. R. S. Liptzer and A. N. Shiryaev, Statistics of Stochastic Processes, Nauka, Moscow (1974), in Russian.

8. P. A. Meyer, Probability and Potentials, Braisdell, Waltham, Mass., 1966.

9. W. J. Park, A multiparameter Gaussian Process, Ann. Math. Stat., 41 (1970), 15821595.

10. S. Watanabe, Stochastic Differential Equations, Sangyo-Tosho, Tokyo (1975), in Japanese.

11. E. Wong and M. Zakai, Martingales and stochastic integrals for processes with a multi-dimensional parameter, Z. Wahrscheinlichkeitstheorie und Verw. Gebiete, 29 (1974), 109-122.

12. J. Yeh, Wiener measure in a space of functions of two variables, Trans. Amer. Math. Soc., 95 (1960), 433-450.

13. G. J. Zimmerman, Some sample function properties of the two-parameter Gaussian process, Ann. Math. Stat., 43 (1972), 1235-1246.

Received September 2, 1980.

UNIVERSITY OF CALIFORNIA

IRVINE, CA 92717 



\section{PACIFIC JOURNAL OF MATHEMATICS}

\section{EDITORS}

DONALD BABBITT (Managing Editor)

University of California

Los Angeles, California 90024

Hugo RossI

University of Utah

Salt Lake City, UT 84112

C. C. MOORE and ANDREW OGG

University of California

Berkeley, CA 94720

\section{J. DugundJI}

Department of Mathematics University of Southern California Los Angeles, California 90007

R. Finn and J. Milgram Stanford University

Stanford, California 94305

\section{ASSOCIATE EDITORS}
R. ARENS
E. F. BeCKENBACH
B. H. Neumann
F. WoLF
K. YoSHIDA

\section{SUPPORTING INSTITUTIONS}

UNIVERSITY OF ARIZONA

UNIVERSITY OF BRITISH COLUMBIA

CALIFORNIA INSTITUTE OF TECHNOLOGY

UNIVERSITY OF CALIFORNIA

MONTANA STATE UNIVERSITY

UNIVERSITY OF NEVADA, RENO

NEW MEXICO STATE UNIVERSITY

OREGON STATE UNIVERSITY
UNIVERSITY OF OREGON

UNIVERSITY OF SOUTHERN CALIFORNIA

STANFORD UNIVERSITY

UNIVERSITY OF HAWAII

UNIVERSITY OF TOKYO

UNIVERSITY OF UTAH

WASHINGTON STATE UNIVERSITY

UNIVERSITY OF WASHINGTON 


\section{Pacific Journal of Mathematics}

\section{Vol. 97, No. $1 \quad$ January, 1981}

Charles A. Asmuth and Joe Repka, Tensor products for $S L_{2}(\mathfrak{k})$. II.

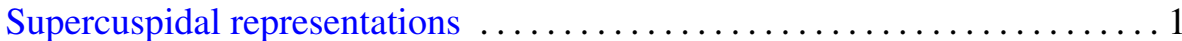

Joseph Barback, On finite sums of regressive isols . ................. 19

Matthew G. Brin and Daniel Russell McMillan, Jr., Generalized

three-manifolds with zero-dimensional nonmanifold set ............29

Kun Soo Chang, Converse measurability theorems for Yeh-Wiener space . . . 59

Christopher Brian Croke, A "maximal torus" type theorem for complete

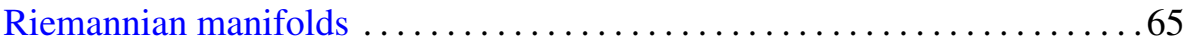

Gustave Adam Efroymson, Sums of squares in planar Nash rings . . . . . . 75

John Robert Fisher, Axiomatic radical and semisimple classes of rings . . . .81

Betty Kvarda, Consecutive integers for which $n^{2}+1$ is composite .......93

Roosevelt Gentry, New diagram proofs of the Hausdorff-Young theorem

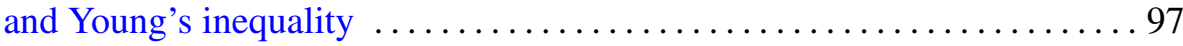

Patrick M. Gilmer, Topological proof of the $G$-signature theorem for $G$

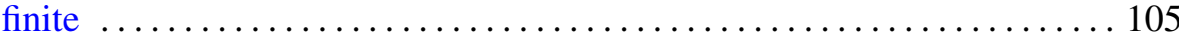

Chung Wei Ha, A noncompact minimax theorem .................. 115

James J. Hebda, Manifolds admitting taut hyperspheres ................ 119

Takayuki Kawada, Sample functions of Pólya processes ............. 125

Peter K. F. Kuhfittig, Common fixed points of nonexpansive mappings by

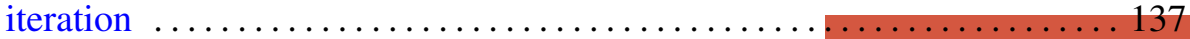

James Thomas Loats and Judith Roitman, Almost rigid Hopfian and dual

Hopfian atomic Boolean algebras .......................... 141

Roger McCann, On embedding semiflows into a radial flow on $l_{2} \ldots \ldots \ldots 151$

John McDonald, Closed orbits of convex sets of operators on the disk

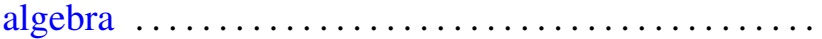

Mark D. Meyerson, Convexity and the table theorem .............. 167

Arnold William Miller, Generic Souslin sets . . . . . . . . . . . . . . . . 171

Takemi Mizokami, On the closed images of paracomplexes $\ldots \ldots \ldots \ldots \ldots 3$

Jagannadham Venkata Pakala and Thomas Stephen Shores, On

compactly packed rings $\ldots \ldots \ldots \ldots \ldots \ldots \ldots \ldots \ldots$

Andrew Pletch, Strong completeness in profinite groups

Wilbur Carrington Whitten, Inverting double knots

James Juei-Chin Yeh, Existence of strong solutions for stochastic

differential equations in the plane 